OPEN ACCESS

Edited by:

Regina Dahlhaus,

University of Erlangen-Nuremberg,

Germany

Reviewed by:

Ka Wan Li,

Vrije Universiteit Amsterdam

Netherlands

Barbara Bardoni,

UMR 7275 Institut de Pharmacologie

Moléculaire et Cellulaire (IPMC),

France

*Correspondence:

Kendal Broadie

kendal.broadie@vanderbilt.edu

Received: 30 October 2017 Accepted: 18 December 2017

Published: 12 January 2018

Citation:

Sears JC and Broadie K (2018) Fragile X Mental Retardation Protein

Regulates Activity-Dependent Membrane Trafficking and Trans-Synaptic Signaling Mediating Synaptic Remodeling.

Front. Mol. Neurosci. 10:440. doi: 10.3389/fnmol.2017.00440

\section{Fragile X Mental Retardation Protein Regulates Activity-Dependent Membrane Trafficking and Trans-Synaptic Signaling Mediating Synaptic Remodeling}

\author{
James C. Sears ${ }^{1}$ and Kendal Broadie ${ }^{1,2,3 *}$ \\ ${ }^{1}$ Department of Biological Sciences, Vanderbilt University, Nashville, TN, United States, ${ }^{2}$ Vanderbilt Kennedy Center for \\ Research on Human Development, Nashville, TN, United States, ${ }^{3}$ Vanderbilt Brain Institute, Vanderbilt University Medical \\ Center, Nashville, TN, United States
}

Fragile $X$ syndrome (FXS) is the leading monogenic cause of autism and intellectual disability. The disease arises through loss of fragile $X$ mental retardation protein (FMRP), which normally exhibits peak expression levels in early-use critical periods, and is required for activity-dependent synaptic remodeling during this transient developmental window. FMRP canonically binds mRNA to repress protein translation, with targets that regulate cytoskeleton dynamics, membrane trafficking, and trans-synaptic signaling. We focus here on recent advances emerging in these three areas from the Drosophila disease model. In the well-characterized central brain mushroom body (MB) olfactory learning/memory circuit, FMRP is required for activity-dependent synaptic remodeling of projection neurons innervating the MB calyx, with function tightly restricted to an earlyuse critical period. FMRP loss is phenocopied by conditional removal of FMRP only during this critical period, and rescued by FMRP conditional expression only during this critical period. Consistent with FXS hyperexcitation, FMRP loss defects are phenocopied by heightened sensory experience and targeted optogenetic hyperexcitation during this critical period. FMRP binds mRNA encoding Drosophila ESCRTIII core component Shrub (human CHMP4 homolog) to restrict Shrub translation in an activity-dependent mechanism only during this same critical period. Shrub mediates endosomal membrane trafficking, and perturbing Shrub expression is known to interfere with neuronal process pruning. Consistently, FMRP loss and Shrub overexpression targeted to projection neurons similarly causes endosomal membrane trafficking defects within synaptic boutons, and genetic reduction of Shrub strikingly rescues Drosophila FXS model defects. In parallel work on the well-characterized giant fiber (GF) circuit, FMRP limits iontophoretic dye loading into central interneurons, demonstrating an FMRP role controlling core neuronal properties through the activity-dependent repression of translation. In the well-characterized Drosophila neuromuscular junction (NMJ) model, developmental synaptogenesis and activity-dependent synaptic remodeling both require extracellular matrix metalloproteinase (MMP) enzymes interacting with the heparan sulfate proteoglycan (HSPG) glypican dally-like protein (DIp) to restrict trans-synaptic 
Wnt signaling, with FXS synaptogenic defects alleviated by both MMP and HSPG reduction. This new mechanistic axis spanning from activity to FMRP to HSPGdependent MMP regulation modulates activity-dependent synaptogenesis. We discuss future directions for these mechanisms, and intersecting research priorities for FMRP in glial and signaling interactions.

Keywords: fragile X syndrome, critical period, signaling, synapse, Drosophila

\section{INTRODUCTION}

Nascent neural circuitry, while functional, is nevertheless still developing and initially manifests activity-dependent refinement and optimization. During early-use critical periods, new neural circuits are highly sensitive to sensory experience, exhibiting a transient window of heightened synaptic remodeling capacity (Hensch, 2004). Sensory input driving downstream circuit activity can result in persistent, long-lasting structural and functional changes, which generally cannot be retrained once the critical period has past (Takesian and Hensch, 2013). During this activity-dependent refinement, excitatory and inhibitory synapses are balanced in circuits, generally by removing excess excitatory synapses and adding new inhibitory synapses, thereby establishing an optimized excitatory/inhibitory (E/I) balance (Doll and Broadie, 2014). Therefore, suitably primed activity-dependent mechanisms must be present to sculpt synaptic connectivity during these critical periods. The fragile $\mathrm{X}$ mental retardation protein (FMRP), which when lost through epigenetic silencing of the FMR1 gene results in fragile $\mathrm{X}$ syndrome (FXS), is a prime candidate for mediating activity-dependent synaptic remodeling during critical periods. FMRP is directly regulated by activity (Weiler et al., 1997; Antar et al., 2004) and, in turn, regulates activity-dependent processes (Huber et al., 2002; Li et al., 2002). Importantly, considerable evidence supports the theory that FXS is caused by excessive excitatory neurotransmission (hyperexcitation theory), reduced inhibitory transmission (hypoinhibition theory), or some combination of both, resulting in an excitation/inhibition imbalance (E/I imbalance theory) (Gibson et al., 2008; Cea-Del Rio and Huntsman, 2014).

The Drosophila FXS disease model has established conserved requirements for Drosophila FMR1 (dfmr1) (Coffee et al., 2010, 2012). Drosophila FMRP has key roles in synaptic remodeling ranging from the larval neuromuscular junction (NMJ) and sensory circuits, to adult circadian clock neurons and the mushroom body (MB) olfactory learning/memory circuitry (Zhang et al., 2001; Pan et al., 2004; Gatto and Broadie, 2009; Gatto et al., 2014). Null dfmr1 mutants display an elevated number of immature synaptic connections in these diverse circuits, as well as the loss of activity-dependent synaptic pruning (Gatto and Broadie, 2008; Tessier and Broadie, 2008). Importantly, Drosophila FMRP is developmentally regulated: FMRP levels are at their highest during very late pupal brain development and the first day of post-eclosion adulthood, with levels then decreasing dramatically at maturity (Tessier and Broadie, 2008). FMRP is required developmentally for synaptogenesis, bouton elimination/pruning, activitydependent refinement and calcium signaling (Gatto and Broadie, 2008, 2009; Tessier and Broadie, 2008, 2011; Doll and Broadie, 2015, 2016). For E/I balance, Drosophila FMRP drives use-dependent down-regulation of synaptic excitability via metabotropic glutamate receptors (mGluRs) (Pan and Broadie, 2007; Pan et al., 2008; Repicky and Broadie, 2008), and promotes GAD levels and GABAergic innervation (Gatto et al., 2014). Given E/I balance is established during the critical period, FMRP loss during this developmental window consistently causes differential activity regulation of excitatory vs. inhibitory neurons in the Drosophila FXS model, with defective activity-dependent synapse morphogenesis and $\mathrm{Ca}^{2+}$ signaling maturation (Doll and Broadie, 2015, 2016).

Fragile X mental retardation protein is an RNA-binding translation repressor (Laggerbauer et al., 2001; Li et al., 2001; Darnell et al., 2011; Ascano et al., 2012; Chen and Joseph, 2015), with translation enhancement also reported (Todd et al., 2003; Muddashetty et al., 2007; Kenny et al., 2014; Fernandez et al., 2015; Kenny and Ceman, 2016). Primary established targets of repression include cytoskeletal and signaling regulators (Zhang et al., 2001, 2005; Lee et al., 2003; Bongmba et al., 2011; Santoro et al., 2012; Friedman et al., 2013; Majumder et al., 2016). Genetic and pharmacological correction of protein levels or elevated signaling in FXS models can rescue synaptic defects. For example, FMRP binds the mRNA encoding microtubule-associated protein IB (MAPIB)/Futsch, dfmr1 null animals overexpress Futsch, and genetic Futsch reduction corrects synaptic structure/function defects in the Drosophila FXS model (Zhang et al., 2001). Likewise, FMRP binds the mRNA encoding actin-binding Profilin/Chickadee to suppress Chickadee levels, with Chickadee overexpression phenocopying $d f m r 1$ null defects, and decreasing Chickadee levels correcting synaptic defects (Reeve et al., 2005). In signaling, Drosophila FMRP genetically interacts with a mGluR in a bidirectional mechanism controlling ionotropic glutamate receptor (iGluR) classes to regulate synaptic function (Pan and Broadie, 2007; Repicky and Broadie, 2008). Drosophila FMRP also limits two heparan sulfate proteoglycan (HSPG) co-receptors that modulate trans-synaptic signaling, and genetic reduction of these HSPGs suppresses synaptic structure/function defects in the Drosophila FXS model (Friedman et al., 2013). Thus, FMRP targets regulating cytoskeletal and signaling dynamics are causally related to synaptic defects characterizing the FXS disease state. The discovery/ordering of such targets is critical for understanding the FXS disease state. 
Mouse and Drosophila FXS models have been utilized to discover and test targets for therapeutic intervention. For example, inhibition of GSK3 $\beta /$ Shaggy with lithium has mediated promising effects (Klein and Melton, 1996; Stambolic et al., 1996; McBride et al., 2005; Choi et al., 2010; Mines and Jope, 2011). Therapeutic targets regulating the cytoskeleton have long been a focus of FXS model tests. For example, FMRP translationally represses Rac1, and Rac1 activity is elevated in FXS models (Lee et al., 2003; Bongmba et al., 2011; Majumder et al., 2016). Importantly, many Rac1 inhibitors are known which may hold therapeutic potential for FXS treatments (Tejada-Simon, 2015). Downstream of Rac1, inhibition of p21-activated kinase (PAK) signaling can prevent phenotypes in the mouse FXS disease model (Dolan et al., 2013). Classic work showed FXS patient-derived cells have reduced cAMP levels and induction (Berry-Kravis and Huttenlocher, 1992; Berry-Kravis et al., 1995). Mouse and Drosophila FXS models similarly show reduced cAMP levels, with Forskolin stimulation of cAMP production significantly diminished, and genetic/pharmacological correction of cAMP levels preventing FXS phenotypes (Kelley et al., 2007; Kanellopoulos et al., 2012). As a final example, the MMP-9 inhibitor minocycline has been shown in mouse and Drosophila FXS models to correct FXS phenotypes (Bilousova et al., 2008; Siller and Broadie, 2011). These strategies highlight mechanisms causally involved in FXS, with the recurrent theme of efficacious inhibition of targets hyper-activated in the disease state. Further investigation of these core pathways in FXS patients and models will likely lead to future clinically relevant discoveries.

Fragile $\mathrm{X}$ mental retardation protein plays key roles in the regulation of intercellular interactions governing synaptic remodeling, including trans-synaptic signaling and glial pruning. Work over the last several years has established that FMRP regulates trans-synaptic signaling at the Drosophila NMJ model synapse, particularly in the control of the founding Wnt Wingless (Wg) signaling pathway (Siller and Broadie, 2011; Friedman et al., 2013). Wg trans-synaptic signaling regulates activity-dependent synaptic structure/function remodeling (Ataman et al., 2008), with the Wg secreted from synapse-associated glia selectively regulating post-synaptic assembly and transmission strength (Kerr et al., 2014). Activity-dependent Wg signaling occurs in a very rapid time frame; for example, the Wg-driven formation of nascent presynaptic boutons ("ghost boutons") occurs within minutes of stimulation (Ataman et al., 2008). Wg trans-synaptic signaling is modulated by extracellular HSPGs [e.g., dally-like protein (Dlp)] and matrix metalloprotease (MMP) enzymes that co-regulate each other in the synaptomatrix surrounding synaptic boutons (Dear et al., 2016). Importantly, HSPG/MMP levels and Wg signaling are altered in parallel in $d f m r 1$ null animals, and the genetic reduction of Dlp, or genetic/pharmacological reduction of secreted MMP1, both correct Drosophila FXS disease model phenotypes (Siller and Broadie, 2011; Friedman et al., 2013). In addition to the above glial involvement in trans-synaptic signaling, glia have also been implicated in neural phagocytosis pruning during remodeling (Tasdemir-Yilmaz and Freeman, 2014). Thus, glia may play central roles during FMRP-dependent synaptic refinement in response to activity states and intercellular signaling cues.
In this review, we focus on recent Drosophila FXS model studies of FMRP in activity-dependent synaptic remodeling. We highlight roles in a range of disparate neural circuits: (1) the adult central brain MB learning/memory circuit during an early-use critical period (Guven-Ozkan and Davis, 2014), (2) the adult giant fiber (GF) escape circuit connecting sensory input to motor output (Boerner and Godenschwege, 2010), and (3) the larval NMJ glutamatergic model synapse (Harris and Littleton, 2015). We concentrate on recent 2017 papers assaying different facets of FMRP biology in these circuits. In the MB circuit, FMRP functions in an activity sensor mechanism to mediate sensory experience refinement of olfactory projection neuron synapses during an early-use critical period, with loss of FMRP resulting in a hyper-excited state that is phenocopied in wildtype animals with intense stimulation (Doll et al., 2017). FMRP suppresses translation of ESCRTIII core component Shrub to enable endosomal membrane trafficking required for critical period activity-dependent synaptic refinement (Vita and Broadie, 2017). In the GF circuit, FMRP limits small molecule permeation in central interneurons, which is disrupted in the Drosophila FXS model (Kennedy and Broadie, 2017). At the NMJ, activity regulates extracellular HSPG/MMP co-localization in the synaptomatrix, within a FMRP-dependent mechanism driving synaptic remodeling (Dear et al., 2017). We end by discussing future directions stemming from this work, as well as emerging avenues on cAMP signal transduction, cytoskeleton regulation, glial-dependent refinement and activity-dependent trans-synaptic signaling impacting the FXS disease state.

\section{FMRP REQUIREMENTS IN CRITICAL PERIOD ACTIVITY-DEPENDENT SYNAPTIC REMODELING}

The Drosophila MB olfactory learning and memory circuit in the developing adult brain has numerous advantages for researching critical periods. With a particularly well-defined neural circuitry map, coupled to a host of genetic tools and transgenic markers, we can probe the mechanisms of activity-dependent remodeling in individually identified single neurons (Figure 1). Olfactory sensory experience can be manipulated in developmental time periods, or different neurons within the defined circuit targeted with bidirectional optogenetics or transgenic toxins, to dissect activity-dependent remodeling in this rapidly developing animal model. In this defined neural circuit, olfactory sensory neurons (OSNs) expressing the same odorant receptor converge on fully mapped antennal lobe (AL) synaptic glomeruli to innervate central brain projection neurons (PNs; Figure 1). PNs output information to the MB calyx by synapsing on Kenyon cells (KCs) involved in learning acquisition and memory consolidation (Figure 1). Using KC clonal analyses, we first discovered that FMRP is required for activity-dependent synaptic pruning downstream of olfactory sensory experience, and in response to targeted optogenetic depolarization (Tessier and Broadie, 2008). Sensory experience and activity both promote FMRP expression, with FMRP levels elevated during late pupariation and the first day post-eclosion (1 dpe), but much lower at 


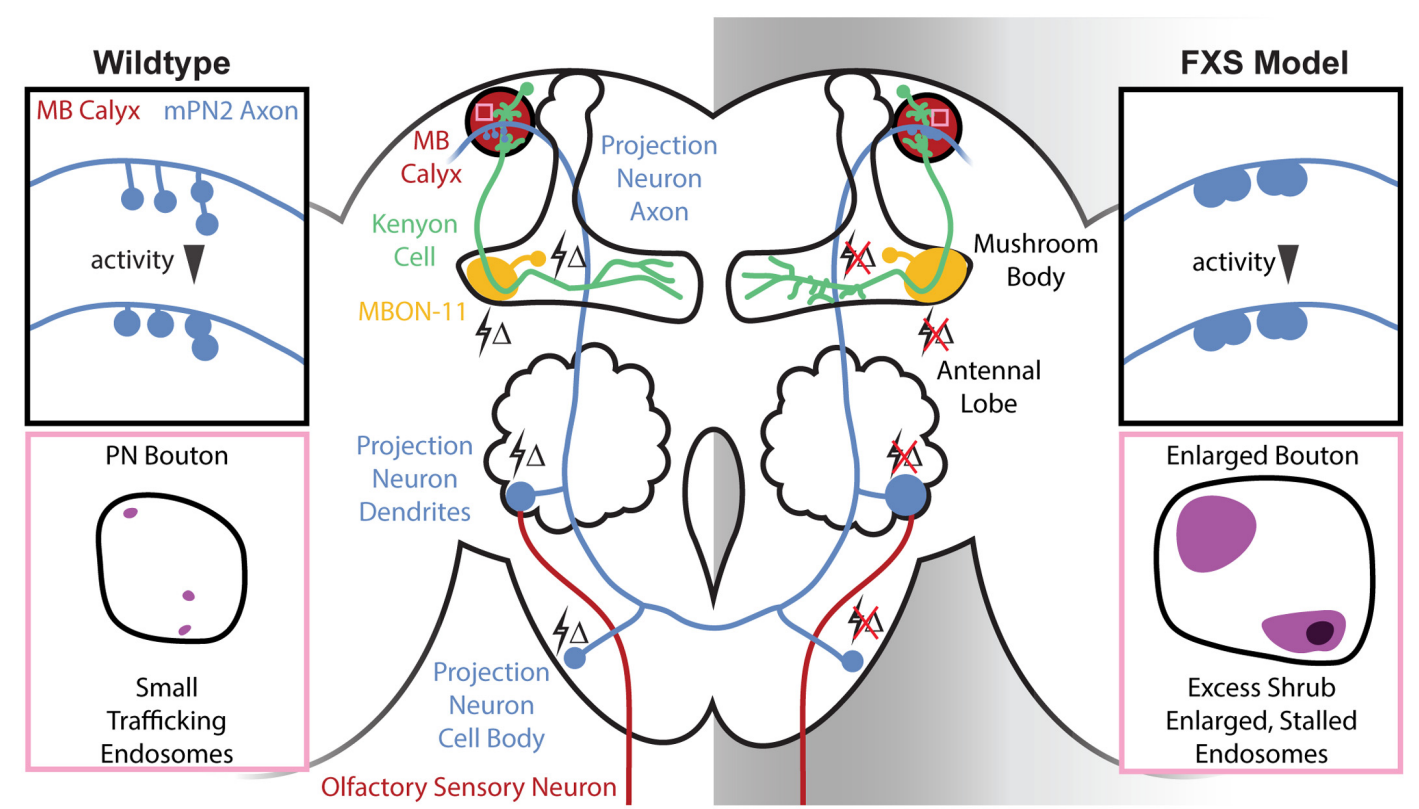

FIGURE 1 | Central brain mushroom body (MB) circuit defects in the Drosophila Fragile X syndrome (FXS) model. Schematic of the Drosophila central brain olfactory circuitry comparing wildtype (Left) and the FXS disease model (Right). Olfactory sensory neurons (OSNs) (red, bottom) expressing specific odorant receptors converge in antennal lobe (AL) glomeruli to synapse on projection neurons (blue, middle). Projection neurons output to the MB calyx (red, top) to synapse on Kenyon cells (KCs) (green), which in turn project to MB axonal lobes to synapse on MB output neurons [e.g., MB output neuron type 11 (MBON-11, yellow)]. Changes in olfactory sensory experience (lightning bolts $\Delta$ ) drive activity-dependent synaptic remodeling throughout this circuit in the early-use critical period, which fails in the FXS condition. Top insets (black boxes): schematic of MB calyx in wildtype and the FXS model. Projection neuron synaptic termini are normally subject to activity-dependent remodeling, but this is absent in the FXS model. The resulting collapsed synaptic architecture with enlarged boutons is phenocopied with strong activity in wildtype. Bottom insets (pink boxes): schematic of single projection neuron synaptic boutons in the wildtype and FXS model MB calyx. The endosomal sorting complex required for transport III (ESCRTIII) core component Shrub normally mediates rapid endocytic membrane trafficking within the PN synaptic boutons, but the FXS model displays an increased number of trafficking-arrested, enlarged synaptic endosomes.

maturity (e.g., 7 dpe). During this transient window, FMRP represses overall protein levels as well as specific FMRP targets (e.g., Profilin/Chickadee; Tessier and Broadie, 2008). This work established an FMRP-defined critical period in the MB circuit for early-use, activity-dependent circuit refinement.

The recent emergence of new transgenic driver libraries allows for an unprecedented, circuit-level investigation of FMRP requirements during this critical period development (Jenett et al., 2012). These new generation, highly selective drivers allow neuron-specific visualization and optogenetic manipulation [e.g., excitatory olfactory PN type 2 (mPN2) and inhibitory $\mathrm{MB}$ output neuron type 11 (MBON-11); Aso et al., 2014; Ito et al., 2014]. Using these tools, the initial goal was to characterize activity-dependent synaptic remodeling during critical period development, and to test for FMRP requirements in this mechanism. In line with the excitatory vs. inhibitory neuron class (Figure 1), targeted optogenetic depolarization results in decreased dendritic size in mPN2 and opposite increase in dendritic arborization in MBON-11 neurons (Doll and Broadie, 2015). Consistently, prevention of depolarization through optogenetic hyperpolarization results in increased mPN2 dendritic arbors and a decrease in MBON11 dendritic size. FMRP loss results in increased dendritic arborization in both neuron classes, and prevents activitydependent remodeling due to either hyper- or hypo-polarization
(Doll and Broadie, 2015). Crucially, these activity-dependent changes normally only occur during the early-use critical period (0-1 dpe), and FMRP is necessary only during this window for synaptic remodeling (Doll and Broadie, 2015). Therefore, neurons without FMRP cannot respond to activity, eliminating their capacity to be refined during circuit optimization (Figure 1). The wider implication of this insensitivity is that FXS disease state neurons are no longer able to mature based on critical period experience in order to fine-tune behavioral responses.

Most critical period activity-dependent refinement studies in this FXS model have been restricted to structural analyses. The one exception is testing the maturation of calcium signaling dynamics with transgenic GCaMP reporters (Doll and Broadie, 2016). In the same excitatory input mPN2 and inhibitory output MBON-11 neuronal pair (Figure 1), dfmr1 null mPN2 shows strongly elevated depolarization-induced $\mathrm{Ca}^{2+}$ transients, whereas MBON-11 manifests an opposite $\mathrm{Ca}^{2+}$ signaling depression during the critical period (Doll and Broadie, 2016). As above with architecture, these functional phenotypes are restricted to the $0-1$ dpe critical period window, with activity-dependent $\mathrm{Ca}^{2+}$ transients largely normalized to wildtype levels in both neuron classes by maturity (e.g., 7 dpe). Excitatory mPN2s manifest a persistent functional defect, with depolarization-induced $\mathrm{Ca}^{2+}$ transients shifted from elevated in the critical period to slightly depressed at maturity (Doll and 
Broadie, 2016). Importantly, cell-specific rescue of FMRP in the critical period restores $\mathrm{Ca}^{2+}$ signaling in both neuron classes, while conditional RNAi knockdown of FMRP phenocopies the dfmr 1 null defects, proving a cell-autonomous, critical period role for FMRP in $\mathrm{Ca}^{2+}$ signaling control (Doll and Broadie, 2016). In wildtype animals, targeted optogenetic depolarization during the critical period entrains increased $\mathrm{Ca}^{2+}$ transients in both neuron classes, but this activity-dependent plasticity is lost in the FXS model, with a slight timing delay in $d f m r 1$ null MBON-11 neurons (Doll and Broadie, 2016). These results suggest an E/I imbalance mechanism: excitatory neurons do not mature due to hyper-excitability, while inhibitory neurons do not mature due to hypo-excitability.

Most recently, mPN2 connectivity in the MB calyx learning/memory center was tested for FMRP requirements in activity-dependent remodeling (Doll et al., 2017). In these well-defined synapses, mPN2 axons project collateral branches with boutons into synaptic microglomeruli innervating $\mathrm{KC}$ dendrites (Figure 1). FMRP regulates mPN2-KC connectivity specifically during the critical period, with branch length decreased and synaptic bouton area increased in dfmr1 nulls (Doll et al., 2017), causing a much more compact innervation pattern (Figure 1). In the mutants, mPN2 microglomeruli display a loss of the presynaptic active zone scaffold Bruchpilot during the critical period, suggesting delayed synaptogenesis (Doll et al., 2017). All defects are completely restricted to the critical period, with normal synaptic architecture and molecular differentiation restored by maturity (e.g., 7 dpe). GFP reconstitution across synaptic partners (GRASP) to test mPN2-KC connections (Feinberg et al., 2008; Pech et al., 2013) reveals that $d f m r 1$ null synaptic contacts are fewer in number, larger in size and more spatially restricted in the critical period, but not at maturity (Figure 1; Doll et al., 2017). EM ultrastructural analysis confirms that synaptic bouton size is increased in $d f m r 1$ null mutants during the critical period. Moreover, directly visualized T-bar synaptic active zones are drastically reduced in density in the FXS model, consistent with the loss of Bruchpilot labeling during the critical period (Doll et al., 2017). Given the activitydependent remodeling during the normal critical period, and the activity insensitivity of $d f m r 1$ mutants, it was hypothesized that connectivity defects arise from activity-dependent refinement that occurs only in wildtype animals.

Odor response mapping studies demonstrate that IR75d OSNs respond to pyrrolidine upstream of $\mathrm{mPN} 2$ (Figure 1; Silbering et al., 2011; Münch and Galizia, 2016). Pyrrolidine exposure in the critical period, but not at maturity, phenocopies $d f m r 1$ synaptic defects and no changes occur in $d f m r 1$ mutants, demonstrating that FMRP is required for sensory experience synaptic remodeling (Doll et al., 2017). At maturity, pyrrolidine exposure causes no changes in wildtype animals, but does cause a reduction in $d f m r 1$ branch length, consistent with a shifted critical period. Optogenetic stimulation during the critical period also results in mPN2-KC connectivity changes in controls, but not dfmr1 mutants (Doll et al., 2017). Conversely, targeted optogenetic hyperpolarization or tetanus toxin neurotransmission blockade both result in the opposite consequence of expanded MB calyx innervation in controls, but not $d f m r 1$ mutants (Figure 1). All manipulations show FMRP is required for activity-dependent synaptic remodeling in the critical period. One exception is hyperpolarization causes partial rescue of $d f m r 1$ bouton area, which may indicate an inhibitory mechanism that can still promote some synaptic refinement despite FMRP loss and decreased GABAergic function in the FXS model (Gatto et al., 2014). Indeed, GABA agonists can rescue hyperexcitation in FXS models (Chang et al., 2008; OlmosSerrano et al., 2010), and activating inhibitory neurons can rescue the experience-driven remodeling (Fagiolini and Hensch, 2000; Hensch, 2004). This may provide a parallel to FMRP critical period requirements, where a weakened inhibitory influence might suppress critical period hyperexcitation in the FXS disease state.

Taken together, these new studies show a transient requirement for FMRP during the early-use sensory experience critical period of synaptic remodeling (Doll et al., 2017). The Drosophila FXS disease model presents synaptic connectivity characteristics replicated by strong developmental activation of the brain circuitry. We conclude, therefore, that FXS is a hyperactivated state, or responsive as if hyper-activated, and that FMRP normally functions in an activity-dependent mechanism to enable circuit refinement during the critical period (Doll et al., 2017). Given the developmental and activity-dependent regulation of FMRP, coupled to its maintained requirement in learning and memory, it is tempting to speculate that loss of FMRP only during this transient window results in persistent network defects at multiple levels, including hyperactivity and improper connectivity (Pan et al., 2004; McBride et al., 2005; Bolduc et al., 2008; Tessier and Broadie, 2008; Doll and Broadie, 2015, 2016; Doll et al., 2017). Moreover, the appearance of a shifted critical period (Doll et al., 2017) is consistent with the argument that delays and developmental perturbations during neural circuit and E/I refinement may result in persistent behavioral abnormalities (Harlow et al., 2010; Takesian and Hensch, 2013). While our current metrics indicate rectification of structural and functional defects following the critical period (Bureau et al., 2008; Doll et al., 2017), there is also apparent overcorrection and blunted calcium signaling at maturity (Tessier and Broadie, 2008; Doll and Broadie, 2016). Future work needs to dissect both transient critical period and lasting mature consequences of FMRP loss in the FXS brain circuitry.

\section{FMRP ROLE IN ENDOCYTIC MEMBRANE TRAFFICKING DURING SYNAPTIC REFINEMENT}

Fragile $\mathrm{X}$ mental retardation protein acts primarily as an mRNA-binding translation suppressor, so this function was explored to test mechanisms of activity-dependent critical period synaptic remodeling (Vita and Broadie, 2017). A Drosophila brain developmental proteomics screen was done to identify candidate protein changes occurring during the critical period window (Tessier and Broadie, 2012). A secondary screen tested for activity-regulated proteins, consistent with a role in developmental plasticity. Finally, candidate hit overexpression 
was assayed for predicted phenocopy of FXS defects, and protein level correction tested for predicted rescue of $d f m r 1$ null phenotypes during critical period development. A new FMRP target meeting all requirements is endosomal sorting complex required for transport III (ESCRTIII) core member Shrub (Vita and Broadie, 2017), Drosophila homolog of yeast Snf7/Vsp32 and human CHMP4 (Babst et al., 2002). Shrub exists as autoinhibited monomers in the cytosol, which assemble in linear polymer arrays of spiral/helical filaments on membranes to drive inverse membrane budding (Teis et al., 2008). ESCRTIII mediates both plasma membrane and organelle trafficking (e.g., endosome-to-multivesicular body; MVB) in cooperation with other ESCRTs and the AAA-ATPase Vps4 (Henne et al., 2013). Canonically, ESCRTIII sorts ubiquitinated proteins to the lysosomal degradation pathway to remove targeted cell surface receptors (Sorkin, 1998; Babst et al., 2002). Importantly, ESCRTIII components are carefully regulated in endosome to MVB maturation, with loss or gain of ESCRTIII components resulting in similar trafficking aberrations, often in the form of greatly enlarged endosomal organelles (Teis et al., 2008).

In Drosophila, Shrub is necessary for developmental axonal pruning, as well as for limiting dendritic arborization (Sweeney et al., 2006). These precedents support a role for Shrub downstream of FMRP translational suppression in synaptic remodeling. A key distinction is that Shrub levels are elevated in the FXS model (Vita and Broadie, 2017), predicting defects caused by excess Shrub. Consistent with selective involvement in the critical period, Shrub levels are elevated in $d f m r 1$ null brains during the $0-1$ dpe window defined above, and FMRP expression rescues Shrub levels during this period (Vita and Broadie, 2017). Importantly, optogenetic stimulation drives increased Shrub levels in wildtype animals during the critical period, whereas dfmrl mutants display no Shrub protein level changes, indicating FMRP mediates activity-dependent regulation. Employing RNA immunoprecipitation, it was found that FMRP binds shrub mRNA (Vita and Broadie, 2017). Taken together, these results demonstrate FMRP limits Shrub levels during the critical period by repressing translation in an activity-dependent mechanism (Vita and Broadie, 2017). With a restricted PN driver (Nrv3-Gal4) for projection neurons innervating the MB calyx (Figure 1), it was shown that Shrub overexpression and FMRP loss similarly cause overelaborated synaptic contacts during the critical period (Vita and Broadie, 2017). Moreover, EM ultrastructural analyses revealed Shrub overexpression and FMRP loss both result in enlarged PN synaptic boutons within the MB calyx (Figures 1, 2). These results confirmed the importance of Shrub elevation in FXS phenotypes, and suggested that endocytic membrane trafficking is required for critical period synaptic refinement.

As a first step in assaying membrane trafficking, the endosome marker Rab5 was assayed in PN synaptic boutons innervating the MB calyx (Vita and Broadie, 2017). Both Shrub overexpression and FMRP loss result in an elevated number of enlarged Rab5-positive endosomes in PN synaptic boutons during the critical period (Figures 1, 2). Consistently, ultrastructural analyses reveal strikingly enlarged endosomic vacuoles within PN synaptic boutons in both the Shrub overexpressing and dfmr1 null animals (Vita and Broadie, 2017). Interestingly, both conditions also display an increased number of enlarged endosomal intraluminal vesicles, consistent with reports of Sfn7 overexpression and interpreted as a consequence of stalled MVB sorting (Teis et al., 2008). Taken together, these results suggest gain of Shrub or loss of FMRP similarly causes traffickingarrested synaptic endosomes (Figures 1, 2). To definitively test the FMRP/Shrub interaction in the context of the FXS disease model, Shrub levels were corrected (shrub/+ heterozygotes) in an otherwise dfmr1 null mutant (Vita and Broadie, 2017). This correction rescues dfmrl phenotypes, with a significant restoration of PN innervation and synaptic bouton area, and complete rescue of endosome trafficking (Figure 2). This work establishes Shrub as an activity-dependent synaptic refinement protein, negatively regulated by FMRP during the critical period to mediate appropriate early-use neural circuit remodeling (Vita and Broadie, 2017). The mechanism likely involves Shrub-dependent endocytic trafficking, either of membrane being internalized during synaptic pruning, or in control of surface guidance molecules regulating activity-dependent synapse elimination (Figure 2).

It is tempting to speculate that stalled MVB maturation is a crucial determinant of the arrested critical period synaptic refinement characterizing the FXS disease state, operating via short-term plasma membrane and/or long-term signaling misregulation (Vita and Broadie, 2017). Evidence for the latter hypothesis comes from developmental pruning studies showing that reduction of cell adhesion molecule Neuroglian coincides with ESCRT-mediated pruning of sensory neuron dendrites during metamorphosis (Zhang et al., 2014). However, Neuroglian levels have not yet been demonstrated to be changed in the Drosophila FXS model, and Neuroglian is not known to be involved in MB synaptic pruning (Reeve et al., 2005; Zhang et al., 2014). Given Shrub is elevated with neuronal activity, we hypothesize it acts to sort activity-dependent reduction of as yet unidentified surface receptors regulating synaptic refinement (Vita and Broadie, 2017). One intriguing possibility is that Shrubmediated membrane trafficking regulates cell surface signals for glial phagocytosis driving synaptic pruning during the earlyuse critical period (Figure 2). Consistently, dfmrl null mutants display delayed developmental MB gamma neuron pruning mediated by phagocytic glia and delayed glial engulfment of damaged axons, as well as clear deficiencies in immune cellmediated engulfment (Tasdemir-Yilmaz and Freeman, 2014; O'Connor et al., 2017). Therefore, we hypothesize that activitydependent defects in synaptic refinement in the FXS disease state could be due to improper intercellular interactions between neurons and glia (Figure 2), which depend on FMRP regulation of Shrub-mediated membrane trafficking.

Shrub misregulation is not the only aberrant translational repression in FXS, and there are broad consequences to neuron properties. Indeed, new evidence suggests this defect alters how molecules permeate dfmr1 null neurons (Kennedy and Broadie, 2017). Iontophoresis of small polar dyes (e.g., neurobiotin, lucifer yellow) has long been used to assay gap junctions linking electrically coupled neurons (Lapper and Bolam, 1991; Hanani, 2012; Kudumala et al., 2013; Lee and Godenschwege, 2015), whereas large dyes (e.g., dextran-tetramethylrhodamine) 

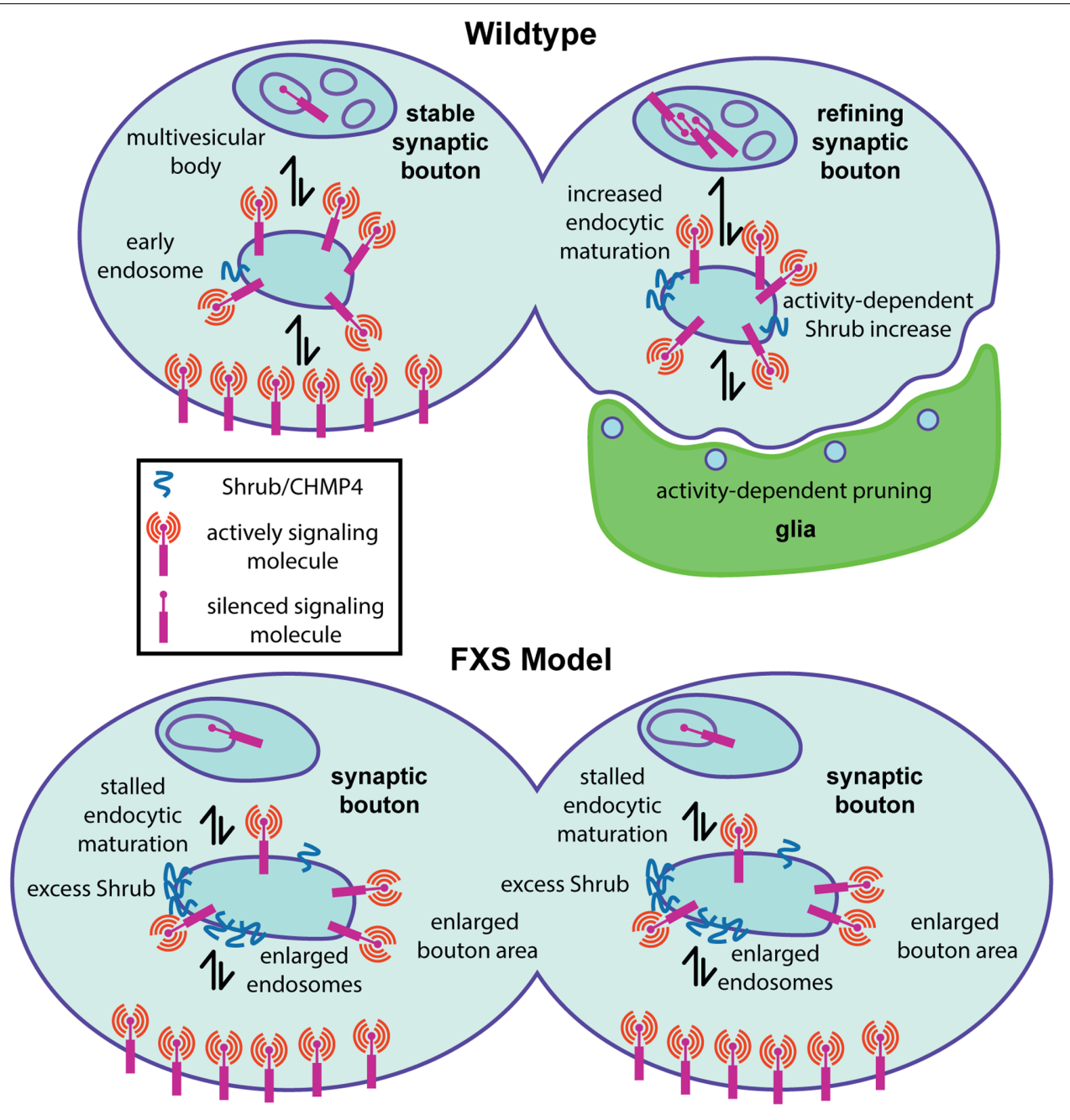

FIGURE 2 | Presynaptic endosomal membrane trafficking defects in the Drosophila FXS model. Diagram summarizing a new fragile X mental retardation protein (FMRP) role in the regulation of presynaptic membrane trafficking by the ESCRTIII core component Shrub/CHMP4. (Top) In wildtype animals, appropriate Shrub levels mediate endosomal membrane trafficking within presynaptic boutons, which is required for activity-dependent synaptic pruning/refinement. It is hypothesized that activity-dependent endosomal trafficking regulates the presentation of surface signaling molecules that trigger phagocytosis by glia (green) during the early-use critical period. (Bottom) In the FXS disease model, excess Shrub translation leads to stalled endosomal membrane trafficking defects, resulting in enlarged endosomes within presynaptic boutons. It is hypothesized that impaired membrane signaling regulation via inappropriate presentation of surface cues driving glial phagocytosis prevents appropriate activity-dependent synaptic pruning/refinement.

fill single neurons without transfer (Phelan et al., 1996). The electrically coupled Drosophila giant fiber interneuron (GFI) transmitting information from sensory neuron inputs to motor neuron outputs (Allen et al., 1998) has been used extensively for such dye injection studies (Boerner and Godenschwege, 2010). Null $d f m r 1$ mutants have strong defects in GFI-dependent behaviors (Martinez et al., 2007), and was therefore targeted for studies of electrical and chemical synaptic connectivity in our FXS model. However, a surprising discovery was made; mutant GFI axons, dendrites and cell bodies are much more easily dye-loaded (Kennedy and Broadie, 2017). The striking defect is specific to small polar dyes, but cannot be attributed to altered electrical synapse coupling. FMRP is absolutely required, since neuron-targeted FMRP fully rescues defects. Membrane properties do not account for the difference, which is due to a highly elevated rate of cytosolic dye incorporation (Kennedy and Broadie, 2017). Our working hypothesis is that elevated protein levels caused by loss of FMRP translational suppression fundamentally alters the cytosolic milieu, to change molecular diffusion rates in FXS model neurons.

\section{FMRP REQUIREMENT IN ACTIVITY-DEPENDENT PROTEOLYTIC SYNAPSE REMODELING}

Up to this point, we have focused on cell-autonomous FMRP requirements, yet a crucial aspect of synaptogenesis and synaptic refinement is coordinated, trans-synaptic signaling between partners (Barros et al., 2011; Dani and Broadie, 
2012). This highly dynamic intercellular communication influences innervation patterns, synaptic architecture and neurotransmission strength, although roles in activity-dependent mechanisms are less clear (Barros et al., 2011; Dani and Broadie, 2012). The extensive toolkit available for the Drosophila glutamatergic NMJ model synapse is ideally suited for testing trans-synaptic signaling within activity-dependent mechanisms (Broadie et al., 2011; Harris and Littleton, 2015). Enlarged presynaptic boutons at the NMJ are easily distinguishable from the post-synaptic subsynaptic reticulum (SSR), and numerous genetic tools, markers and assays separate pre- versus post-synaptic requirements (Harris and Littleton, 2015). Signaling ligands must necessarily traverse the extracellular synaptomatrix. Two key synaptomatrix regulatory factors are (1) HSPGs and (2) matrix metalloproteinases (MMPs; Figure 3). HSPGs contain a core protein and heparan sulfate (HS) glycosaminoglycan (GAG) chains, which bind MMPs and extracellular signaling ligands (Park et al., 2000; Tocchi and Parks, 2013). HSPGs also link MMPs to their targets, promoting proteolytic activation/specificity (Tocchi and Parks, 2013). At the Drosophila NMJ, HSPGs regulate synaptic architecture, presynaptic active zone size/number and post-synaptic function, and serve to localize Wnt signaling ligands (Johnson et al., 2006; Dani et al., 2012; Kamimura et al., 2013).

In both mammalian synapses and the Drosophila NMJ model, extracellular MMPs directly and indirectly regulate the transsynaptic signaling ligands modulating synaptic structure and function (Wlodarczyk et al., 2011; Dear et al., 2016). The mammalian genome encodes at least 24 MMPs with reportedly redundant/overlapping functions, many of which are localized to synapses. In contrast, Drosophila MMPs are represented by just two genes, $m m p 1$ and $m m p 2$, which encode a single secreted and single GPI-anchored enzyme, respectively; although an anchored MMP1 has recently been described (Llano et al., 2000, 2002; LaFever et al., 2017). Compared to the MMP complexity in mammals, Drosophila enables reductionist testing of MMP roles in the FXS state. In Drosophila, both MMP1 and MMP2 regulate axonal and dendritic architecture (Kuo et al., 2005; Yasunaga et al., 2010; Depetris-Chauvin et al., 2014). At the Drosophila NMJ, both MMPs limit presynaptic growth, functional differentiation, and Wnt Wg trans-synaptic signaling (Dear et al., 2016). Interestingly, while MMP1 promotes MMP2 and HSPG Dlp localization, MMP2 limits MMP1 and Dlp localization at the synapse (Dear et al., 2016). These interactions suggest a complex level of interplay between MMPs and HSPGs within the synaptomatrix interface. Functionally, MMPs cleave not only extracellular matrix (ECM) targets during axon pathfinding (Miller et al., 2007, 2011), but also cell adhesion molecules (CAMs) in activity-dependent mechanisms sculpting synapse structure, turning off signaling and mobilizing membrane turnover in processes associated with neurological disorders including FXS (Nagappan-Chettiar et al., 2017).

In Wnt Wg signaling, presynaptic activity leads to $\mathrm{Wg}$ secretion, which binds to Frizzled-2 (Fz2) receptors on both pre- and post-synaptic cells (Koles and Budnik, 2012). Wg signaling drives both divergent and non-canonical Wnt cascades in pre- and post-synaptic cells (Figure 3), modulating both synapse structure and function (Koles and Budnik, 2012). Importantly, activity-induced Wg secretion drives activitydependent synaptic remodeling, which operates within a rapid time-frame to promote formation of "ghost boutons"; immature boutons with presynaptic but not post-synaptic specialization (Ataman et al., 2008). Critically, extracellular HSPGs are integrally involved in Wg trans-synaptic signaling (Figure 3), highlighting the importance of the synaptomatrix in $\mathrm{Wg}$ signaling regulation (Harris and Littleton, 2015). Specifically, the secreted HSPG Perlecan balances pre- and post-synaptic Wg signaling by promoting post-synaptic Wg localization (Kamimura et al., 2013). Moreover, the GPI-anchored HSPG Dlp regulates $\mathrm{Wg}$ signaling in a concentration-dependent manner (Figure 3): based on Dlp co-receptor levels relative to Fz2 receptor and Wg ligand, Dlp can either restrict or promote Wg signaling as a negative and positive signaling regulator (Yan et al., 2009). Importantly, FMRP restricts synaptic levels of two HSPGs (Dlp and Syndecan) to regulate Wnt Wg transsynaptic signaling, which is strongly misregulated in the FXS disease model (Friedman et al., 2013). Given the complex interactions between neural activity states, MMP proteolytic function, HSPG coreceptors and signaling mechanisms, activitydependent Dlp-MMP interactions badly needed to be compared in normal versus FXS model synapses (Dear et al., 2017).

To test activity-dependent mechanisms, temperature-sensitive dTRPA1 channels were used to acutely depolarize neurons over a 1-h period (Hamada et al., 2008; Pulver et al., 2009). These studies demonstrated that MMP1, but not MMP2, is required to form ghost boutons (Dear et al., 2017). Consistently, dTRPA1 activation, or high $\left[\mathrm{K}^{+}\right]$depolarization for just $10 \mathrm{~min}$, rapidly increases MMP1 at the synapse (Figure 3). Conversely, MMP2 is reduced by stimulation, as predicted since MMP1 limits MMP2 (Dear et al., 2016). Moreover, stimulated synapses rapidly elevate Dlp, with increased Dlp and MMP1 co-localization (Dear et al., 2017), supporting previous findings of genetic interaction at the NMJ. Importantly, the Dlp-Mmp1 co-localization in synaptic subdomains is significantly increased following acutely elevated neuronal activity in just $10 \mathrm{~min}$ (Figure 3). Since HSPGs are known to anchor proteases in other contexts (Tocchi and Parks, 2013), the dependence of MMP1 localization on Dlp was next tested. Both genetic mutant and targeted RNAi reduction of Dlp reduce synaptic MMP1 levels dramatically, whereas Dlp overexpression causes an opposing MMP1 increase at the synapse (Dear et al., 2017). These results show that the GPIanchored Dlp regulates secreted MMP1 localization (Figure 3). Moreover, overexpression of Dlp lacking HS-GAG chains causes no change in MMP1 localization, suggesting that the HS-GAG chains are necessary for MMP1 synaptic localization (Dear et al., 2017). These results are consistent with other studies that have established roles for HS-GAG chains in HSPG activity at the synapse (Baeg et al., 2001; Johnson et al., 2006; Yan et al., 2009).

Given activity recruits HSPG Dlp, which in turn localizes MMP1 at the synapse, there is expected to be an activitydependent increase in proteolytic activity surrounding synaptic boutons. To test this prediction, a dye-quenched fluorogenic gelatin substrate was tested in in situ zymography studies of protease enzymatic function (Siller and Broadie, 2011; Shilts and 


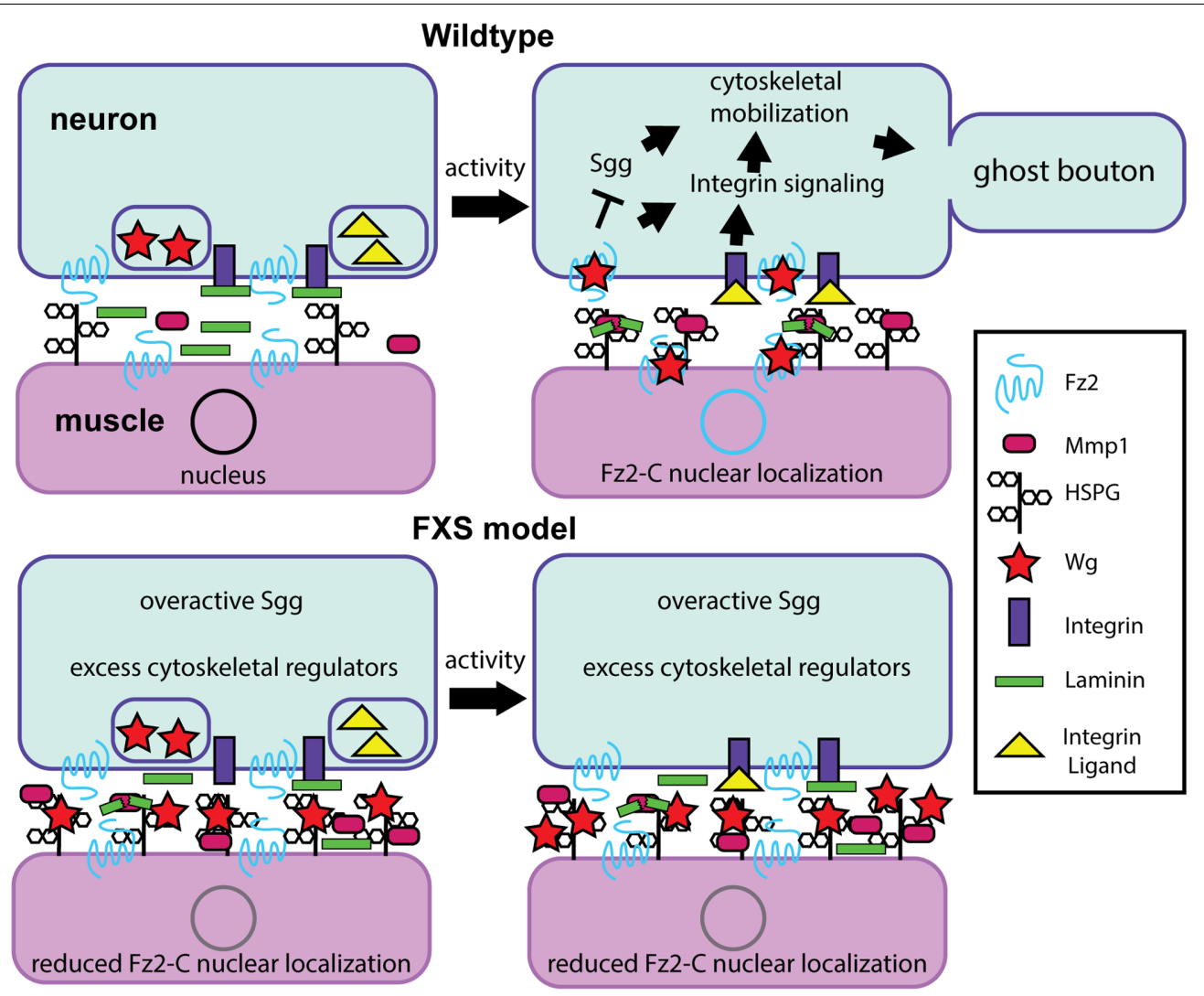

FIGURE 3 | Synaptomatrix trans-synaptic signaling defects in the Drosophila FXS model. Diagram summarizing a new requirement for the secreted matrix metalloproteinase 1 (MMP1) during activity-dependent synaptic remodeling. (Top) In wildtype animals, an activity-dependent FMRP mechanism is required for neural activity to drive heparan sulfate proteoglycan (HSPG) dally-like protein (Dlp) localization at the synapse to recruit MMP1, whose enzymatic function is required for activity-dependent ghost bouton formation. HSPG-MMP1 directed proteolysis drives trans-synaptic Wnt Wingless (Wg) signaling for activity-dependent ghost bouton formation. Activity drives presynaptic signaling via the Frizzled-2 (Fz2) Wg receptor inhibiting GSK3 $\beta /$ Shaggy and integrin receptor signaling to control cytoskeleton dynamics, and post-synaptic Fz2 C-terminal cleavage and subsequent Fz2-C nuclear localization regulating new protein synthesis. It is hypothesized that MMP1 may cleave synaptomatrix Laminin to regulate ligand interactions with integrin receptors. (Bottom) In the FXS disease model, without FMRP DIp and MMP1 are significantly increased at the synapse under basal resting conditions, and their levels do not change with activity manipulations. This activity-insensitivity prevents appropriate activity-dependent regulation of trans-synaptic signaling in the synaptomatrix, likely through inappropriate sequestration of the Wg ligand by HSPG DIp. It is hypothesized that this defect is also linked to improper integrin signaling regulation.

Broadie, 2017). Importantly, Dlp reduction results in reduced MMP-dependent proteolytic activity, while Dlp overexpression, with or without HS-GAG chains, elevates enzymatic function (Dear et al., 2017). These results are consistent with the hypothesis that synaptic Dlp levels tune synaptomatrix MMP1 proteolytic cleavage capacity, possibly via a Dlp core protein interaction resulting in protease activation (Figure 3 ). With the knowledge that basal MMP1 levels are tuned by membraneanchored Dlp, it remained to be tested whether the acute neuronal activity-induced MMP1 increase also depends on Dlp. Indeed, Dlp loss suppresses activity-dependent MMP1 localization at the synapse, whereas Dlp overexpression elevates MMP1 levels and co-localization with Dlp (Dear et al., 2017). In line with above studies, overexpression of a Dlp isoform lacking HS-GAG chains results in a reduced activity-dependent enrichment of MMP1 at the synapse (Figure 3). Importantly, whereas synaptic MMP1 abundance tightly depends on Dlp, loss of MMP1 does not prevent the activity-dependent synaptic enrichment of Dlp (Dear et al., 2017). Thus, synaptic MMP1 localization depends on Dlp, but not vice versa. Taken together, these results support the conclusion that Dlp is absolutely necessary for the rapid activity-dependent synaptic localization of MMP1.

In the Drosophila FXS model, Dlp is constitutively elevated at the NMJ synapse, and reduction of Dlp (or dependent MMP1) in otherwise $d f m r 1$ null mutants suppresses FXS synaptogenic phenotypes (Siller and Broadie, 2011; Friedman et al., 2013). Therefore, activity-dependent Dlp and MMP1 synaptic enrichment was tested in the FXS model (Dear et al., 2017). As expected, MMP1 synaptic localization is strikingly increased in $d f m r 1$ null mutants (Figure 3). One interpretation is that this enrichment reflects a FXS hyper-excited state, manifested in elevated Dlp localization at the synapse (Friedman et al., 2013). Consistent with this idea, genetic reduction of Dlp restores normal MMP1 levels in $d f m r 1$ null synapses (Dear et al., 2017). Importantly, acute stimulation in $d f m r 1$ mutants 
causes no activity-dependent enrichment of MMP1 (Dear et al., 2017), demonstrating that MMP1 is insensitive to activitydependent regulation in the FXS condition (Figure 3). Moreover, Dlp reduction restores activity-induced MMP1 enrichment in dfmr1 null synapses (Dear et al., 2017). Just like stimulated controls, stimulated dfmr1 nulls heterozygous for Dlp display striking synaptic enrichment of Mmp1 (Figure 3). Therefore, Dlp is the critical link determining activity-regulated synaptic MMP1 localization downstream of FMRP. These findings suggest MMP inhibition may ameliorate FXS phenotypes; for example, in the context of mGluR-induced MMP9 hyperactivity (Bilousova et al., 2008). These results also indicate that targeting the misregulated activity-dependent mechanism of Dlp mediating activity-dependent overabundance of synaptic MMP1 could potentially prevent inappropriate connections caused by hyperexcitability in the FXS condition.

This activity-FMRP-HSPG-MMP regulatory mechanism of synapse remodeling presents questions. A key question is the means by which activity-dependent MMP1 localization is restored by reducing Dlp in the FXS model. It is probable that an unidentified synaptomatrix player is involved. Since Dlp can activate and inhibit Wg signaling (Yan et al., 2009; Dani et al., 2012), reducing Dlp could restore proportionality between interacting synaptomatrix components (Figure 3). Altered Dlp sulfation may change protease activity (Tocchi and Parks, 2013), perhaps in concert with other effectors, such as HSPG-sulfating hs6st and sulf1 genes that modulate Wg signaling (Dani et al., 2012). Alternatively, Wg transsynaptic signaling is reduced in the FXS condition (Friedman et al., 2013), and Wg itself may feedback to restore activitydependent MMP1 function (Figure 3). Another possibility is that a synaptomatrix regulator preventing excess Dlp from misregulating activity-dependent MMP1 could be lost in the FXS condition. Indeed, FMRP can promote protein levels (Feng et al., 1997; Derlig et al., 2013), and this could include synaptomatrix proteins. For example, other HSPGs (e.g., Perlecan) might consolidate $\mathrm{Wg}$ signaling, thus restoring a more normal activity-dependent dynamic (Figure 3). Relevant MMP1 catalytic targets are unclear, though it is tempting to speculate secreted MMP1 may cleave Laminin-A to enable activity-dependent integrin signaling (Tsai et al., 2012; Lee et al., 2017). Whatever further elements of the synaptomatrix mechanism have yet to be elucidated, the finding that activitydependent regulation can be restored in the Drosophila FXS disease model opens exciting possibilities for new FXS therapeutic treatments, and may lead to the discovery of novel activity-regulated extracellular molecules critical for synaptic remodeling.

\section{FUTURE DIRECTIONS}

The very recent work discussed in this article highlights the utility of the Drosophila FXS disease model for the study of developmental activity-dependent mechanisms at synaptic connections, during use-dependent synaptic remodeling and in early-use critical periods. These new advances further demonstrate FMRP requirements in activity-dependent regulation of protein translation and control of signaling mechanisms operating at the heart of synapse formation and refinement. The particularly well-characterized central brain MB olfactory learning and memory circuitry has become a powerful vehicle for determining molecular mechanisms disrupted by FMRP loss, cellular mechanisms of activity-dependent synaptic remodeling, and the means of establishing excitatory/inhibitory synapse balance during the critical period (Doll et al., 2017; Vita and Broadie, 2017). In parallel, the classic GF visual escape circuit linking sensory input, brain integration and motor output via particularly large and well-characterized interneurons has the promise of providing an exciting new avenue to dissect FMRP requirements (Kennedy and Broadie, 2017). Finally, the malleable NMJ provides a large and genetically tractable glutamatergic synapse model, which continues to be instrumental in the discovery and elucidation of FMRP synaptic requirements, including intracellular signaling, intercellular interactions, and trans-synaptic pathways that strongly contribute to the FXS disease state (Dear et al., 2017). These diverse circuits will continue to be the focus of future studies, as we seek to determine generalizable FMRP requirements throughout the entire nervous system, as well as selective FMRP roles in specific neural circuits and synapses.

Our current understanding of FMRP requirements during the critical period paves the way for future studies examining molecular mechanisms of activity-dependent refinement. Based on recent findings (Doll and Broadie, 2016), we hypothesize that developmental misregulation of activity-induced $\mathrm{Ca}^{2+}$ signaling is a core contributor to the FXS condition. Importantly, classic memory-linked pathways (e.g., cAMP pathway) connect directly and indirectly to $\mathrm{Ca}^{2+}$ signaling (Davis and Dauwalder, 1991; Skoulakis et al., 1993; Kanellopoulos et al., 2012), with pathway members enriched in Drosophila brain MB and AL (Figure 1; Crittenden et al., 1998). FXS patient-derived cells and models similarly show reduced cAMP levels, and genetic/pharmacological correction of cAMP levels prevents FXS model phenotypes (Berry-Kravis and Huttenlocher, 1992; BerryKravis et al., 1995; Kelley et al., 2007; Kanellopoulos et al., 2012). Downstream of cAMP, PKA phosphorylates a wide range of neuronal targets (Sassone-Corsi, 2012), and enhances excitability in both excitatory and inhibitory neurons to promote activitydependent remodeling (Lee, 2015). A likely downstream target, the small GTPase Racl, acts as a molecular switch in structural and functional synaptic plasticity, and is of interest in the context of FXS hyperexcitability (Lee et al., 2003; Schenck et al., 2003; Bongmba et al., 2011; Goto et al., 2013, 2014; TejadaSimon, 2015). Interestingly, inhibition of PAK downstream of Rac1 prevents FXS model phenotypes (Dolan et al., 2013). We therefore hypothesize that FXS phenotypes associated with aberrant $\mathrm{Ca}^{2+}$-cAMP-PKA-Rac1-PAK signaling likely occur in both the MB and AL during the early-use critical period (Doll et al., 2017; Vita and Broadie, 2017).

We are increasingly aware of possible intercellular interactions in the FXS state, such as neuron-glia roles in circuit refinement (Logan, 2017). Based on our recent work (Vita and Broadie, 2017), we hypothesize dysregulated neuronal surface signaling 
cues impair glia-mediated phagocytosis driving synaptic pruning during activity-dependent refinement (Figure 2). Specifically, we propose disrupted membrane trafficking due to elevated ESCRTIII Shrub levels could alter a surface signal for glial phagocytosis (Vita and Broadie, 2017). Consistently, gliamediated developmental phagocytosis pruning of $\mathrm{MB}$ gamma neuron collateral branches is reduced/delayed in the absence of FMRP (O'Connor et al., 2017). Studies to date have focused primarily on glial clearance via the Draper/Ced-1/MEGF-10 receptor pathway (Musashe et al., 2016; Lu et al., 2017). We propose that FMRP loss may cause improper refinement through signaling defects that prevent glial phagocytosis, delay signaling processes that promote phagocytosis, or manifest aberrations in glial inability to sufficiently regulate or respond to other cells during critical period refinement. Interestingly, glia modulate the excitation/inhibition balance via a GABA uptake mechanism regulating synaptogenesis (Muthukumar et al., 2014). We therefore hypothesize that neuron-glia interactions may also modulate synaptic excitation/inhibition balance in critical period refinement. Recent work in mice shows glial FMRP is necessary, but not sufficient, for FXS model dendritic spine dynamics (Hodges et al., 2017), and co-cultures with astrocytes lacking FMRP illustrate delayed development (Jacobs et al., 2016), indicating that FMRP can act directly in glia as well as in neurons.

In the NMJ model, misregulation of Wnt Wg trans-synaptic signaling is an established cause of FXS phenotypes (Friedman et al., 2013). FMRP is required for activity-dependent HSPG Dlp regulation of extracellular MMP1 synaptic localization and enzymatic function (Dear et al., 2017). MMP1, in turn, is absolutely required for rapid synaptic bouton formation in response to activity. Moreover, Tissue Inhibitor of MMP (TIMP) overexpression prevents synaptic defects in the Drosophila FXS model (Siller and Broadie, 2011), suggesting that synaptomatrix protease regulation is another avenue worth investigating in the FMRP-Dlp-MMP1 pathway (Dear et al., 2017). HSPG Syndecan is negatively regulated by FMRP (Friedman et al., 2013), and may therefore also be involved. Downstream of altered Wnt Wg trans-synaptic signaling, defective Fz2-C nuclear import is well described in the Drosophila FXS disease model (Friedman et al., 2013), but it remains to be tested whether autocrine Wg signaling is also impacted. Based on work showing that inhibition of the Wg divergent canonical target GSK3 $\beta$ /Shaggy is a promising FXS therapeutic treatment (Klein and Melton, 1996; Stambolic et al., 1996; McBride et al., 2005; Choi et al., 2010; Mines and Jope, 2011), we hypothesize that overabundant synaptic Dlp sequesters Wg ligand, inhibiting Wg signaling, and therefore the activitydependent suppression of GSK3 $\beta /$ Shaggy. Future work will test whether Wg sequestration by excess Dlp explains the activityinsensitivity of $d f m r 1$ null synapses. Synapse-associated glia also regulate Wg trans-synaptic signaling (Kerr et al., 2014; Kopke

\section{REFERENCES}

Allen, M. J., Drummond, J. A., and Moffat, K. G. (1998). Development of the giant fiber neuron of Drosophila melanogaster. J. Comp. Neurol. 397, 519-531. doi: 10.1002/(SICI)1096-9861(19980810)397:4<519::AID-CNE5>3.0.CO;2-4 et al., 2017), indicating another plausible source of aberrant synaptomatrix regulation that needs to be explored in the FXS condition.

As we continue ongoing studies exploiting the Drosophila FXS disease model, we posit outstanding needs to dissect developmental activity-dependent synaptic remodeling and connectivity refinement mechanisms, both in the brain and at the NMJ. Within the brain AL-MB olfactory circuit (Figure 1), OSN, PN, and KC synaptic connections are well suited to pursue the mechanisms of trans-synaptic signaling (e.g., Notch, Wg), synaptomatrix regulation (e.g., HSPG, MMP), signal transduction (e.g., cAMP-PKA, actin cytoskeleton), and intercellular interactions (e.g., neuron-glia). This circuit is also ideal for testing mechanisms of excitation/inhibition balance (e.g., mPN2 vs. MBON-11; Figure 1) developing in response to sensory experience during early-use critical periods. Our work highlights a restricted, transient window of FMRP requirement coinciding with peak FMRP levels. In parallel, the NMJ glutamatergic model synapse will be instrumental for investigating the interplay of the multiple bidirectional transsynaptic signaling pathways regulated by an increasingly defined synaptomatrix (Figure 3). This system is also ideal for testing activity-dependent synaptic remodeling mechanisms, including bouton addition and elimination, and glial involvement in the refinement of the pre- and post-synaptic sides of the synapse. In addition, NMJ findings will continue to inform and direct ongoing central brain studies. Our goal is to continue to discover cellular and molecular mechanisms of activity-dependent circuit remodeling that optimize behavioral performance, and to reveal the FMRP-dependent neurodevelopmental processes that go awry in FXS, so as to be able to devise effective new treatments for this devastating disease state.

\section{AUTHOR CONTRIBUTIONS}

JS and $\mathrm{KB}$ co-wrote this article.

\section{FUNDING}

This work was funded by National Institute of Mental Health R01s MH084989/MH096832 to KB.

\section{ACKNOWLEDGMENTS}

The authors are grateful to members of the Broadie Laboratory, especially Mary Lynn Dear and Dominic Vita, for critical input on this article.

Antar, L. N., Afroz, R., Dictenberg, J. B., Carroll, R. C., and Bassell, G. J. (2004). Metabotropic glutamate receptor activation regulates Fragile X mental retardation protein and FMR1 mRNA localization differentially in dendrites and at synapses. J. Neurosci. 24, 2648-2655. doi: 10.1523/JNEUROSCI.009904.2004 
Ascano, M., Mukherjee, N., Bandaru, P., Miller, J. B., Nusbaum, J. D., Corcoran, D. L., et al. (2012). FMRP targets distinct mRNA sequence elements to regulate protein expression. Nature 492, 382-386. doi: 10.1038/nature11737

Aso, Y., Sitaraman, D., Ichinose, T., Kaun, K. R., Vogt, K., Belliart-Guérin, G., et al. (2014). Mushroom body output neurons encode valence and guide memory-based action selection in Drosophila. eLife 3:e04580. doi: 10.7554/eLife. 04580

Ataman, B., Ashley, J., Gorczyca, M., Ramachandran, P., Fouquet, W., Sigrist, S. J., et al. (2008). Rapid activity-dependent modifications in synaptic structure and function require bidirectional Wnt signaling. Neuron 57, 705-718. doi: 10.1016/j.neuron.2008.01.026

Babst, M., Katzmann, D. J., Estepa-Sabal, E. J., Meerloo, T., and Emr, S. D. (2002). Escrt-III: an endosome-associated heterooligomeric protein complex required for mvb sorting. Dev. Cell 3, 271-282. doi: 10.1016/S1534-5807(02)00220-4

Baeg, G. H., Lin, X., Khare, N., Baumgartner, S., and Perrimon, N. (2001). Heparan sulfate proteoglycans are critical for the organization of the extracellular distribution of Wingless. Development 128, 87-94. doi: 10.1016/S1534-5807(02) 00220-4

Barros, C. S., Franco, S. J., and Muller, U. (2011). Extracellular matrix: functions in the nervous system. Cold Spring Harb. Perspect. Biol. 3:a005108. doi: 10.1101/ cshperspect.a005108

Berry-Kravis, E., Hicar, M., and Ciurlionis, R. (1995). Reduced cyclic AMP production in fragile $\mathrm{X}$ syndrome: cytogenetic and molecular correlations. Pediatr. Res. 38, 638-643. doi: 10.1203/00006450-199511000-00002

Berry-Kravis, E., and Huttenlocher, P. R. (1992). Cyclic AMP metabolism in fragile X syndrome. Ann. Neurol. 31, 22-26. doi: 10.1002/ana.410310105

Bilousova, T. V., Dansie, L., Ngo, M., Aye, J., Charles, J. R., Ethell, D. W., et al. (2008). Minocycline promotes dendritic spine maturation and improves behavioural performance in the fragile X mouse model. J. Med. Genet. 46, 94-102. doi: 10.1136/jmg.2008.061796

Boerner, J., and Godenschwege, T. A. (2010). Application for the Drosophila ventral nerve cord standard in neuronal circuit reconstruction and in-depth analysis of mutant morphology. J. Neurogenet. 24, 158-167. doi: 10.3109/01677063.2010. 489624

Bolduc, F. V., Bell, K., Cox, H., Broadie, K. S., and Tully, T. (2008). Excess protein synthesis in Drosophila Fragile X mutants impairs long-term memory. Nat. Neurosci. 11, 1143-1145. doi: 10.1038/nn.2175

Bongmba, O. Y. N., Martinez, L. A., Elhardt, M. E., Butler, K., and TejadaSimon, M. V. (2011). Modulation of dendritic spines and synaptic function by Rac1: a possible link to Fragile X syndrome pathology. Brain Res. 1399, 79-95. doi: 10.1016/j.brainres.2011.05.020

Broadie, K., Baumgartner, S., and Prokop, A. (2011). Extracellular matrix and its receptors in Drosophila neural development. Dev. Neurobiol. 71, 1102-1130. doi: 10.1002/dneu.20935

Bureau, I., Shepherd, G. M. G., and Svoboda, K. (2008). Circuit and plasticity defects in the developing somatosensory cortex of Fmr1 knock-out mice. J. Neurosci. 28, 5178-5188. doi: 10.1523/JNEUROSCI.1076-08.2008

Cea-Del Rio, C. A., and Huntsman, M. M. (2014). The contribution of inhibitory interneurons to circuit dysfunction in Fragile X Syndrome. Front. Cell. Neurosci. 8:245. doi: $10.3389 /$ fncel.2014.00245

Chang, S., Bray, S. M., Li, Z., Zarnescu, D. C., He, C., Jin, P., et al. (2008). Identification of small molecules rescuing fragile $\mathrm{X}$ syndrome phenotypes in Drosophila. Nat. Chem. Biol. 4, 256-263. doi: 10.1038/nchembio.78

Chen, E., and Joseph, S. (2015). Fragile X mental retardation protein: a paradigm for translational control by RNA-binding proteins. Biochimie 114, 147-154. doi: 10.1016/j.biochi.2015.02.005

Choi, C. H., McBride, S. M. J., Schoenfeld, B. P., Liebelt, D. A., Ferreiro, D., Ferrick, N. J., et al. (2010). Age-dependent cognitive impairment in a Drosophila Fragile X model and its pharmacological rescue. Biogerontology 11, 347-362. doi: 10.1007/s10522-009-9259-6

Coffee, R. L., Tessier, C. R., Woodruff, E. A., and Broadie, K. (2010). Fragile X mental retardation protein has a unique, evolutionarily conserved neuronal function not shared with FXR1P or FXR2P. Dis. Model. Mech. 3, 471-485. doi: $10.1242 / \mathrm{dmm} .004598$

Coffee, R. L., Williamson, A. J., Adkins, C. M., Gray, M. C., Page, T. L., and Broadie, K. (2012). In vivo neuronal function of the fragile X mental retardation protein is regulated by phosphorylation. Hum. Mol. Genet. 21, 900-915. doi: $10.1093 / \mathrm{hmg} / \mathrm{ddr} 527$
Crittenden, J. R., Skoulakis, E. M. C., Han, K. A., Kalderon, D., and Davis, R. L. (1998). Tripartite mushroom body architecture revealed by antigenic markers. Learn. Mem. 5, 38-51.

Dani, N., and Broadie, K. (2012). Glycosylated synaptomatrix regulation of transsynaptic signaling. Dev. Neurobiol. 72, 2-21. doi: 10.1002/dneu.20891

Dani, N., Nahm, M., Lee, S., and Broadie, K. (2012). A targeted glycan-related gene screen reveals heparan sulfate proteoglycan sulfation regulates WNT and BMP trans-synaptic signaling. PLOS Genet. 8:e1003031. doi: 10.1371/journal.pgen. 1003031

Darnell, J. C., Van Driesche, S. J., Zhang, C., Hung, K. Y. S., Mele, A., Fraser, C. E., et al. (2011). FMRP stalls ribosomal translocation on mRNAs linked to synaptic function and autism. Cell 146, 247-261. doi: 10.1016/j.cell.2011.06.013

Davis, R. L., and Dauwalder, B. (1991). The Drosophila dunce locus: learning and memory genes in the fly. Trends Genet. 7, 224-229. doi: 10.1016/0168-9525(91) 90369-2

Dear, M. L., Dani, N., Parkinson, W., Zhou, S., and Broadie, K. (2016). Two classes of matrix metalloproteinases reciprocally regulate synaptogenesis. Development 143, 75-87. doi: 10.1242/dev.124461

Dear, M. L., Shilts, J., and Broadie, K. (2017). Neuronal activity drives FMRPand HSPG-dependent matrix metalloproteinase function required for rapid synaptogenesis. Sci. Signal. 10:eaan3181. doi: 10.1126/scisignal.aan3181

Depetris-Chauvin, A., Fernández-Gamba, Á., Gorostiza, E. A., Herrero, A., Castañ;o, E. M., and Ceriani, M. F. (2014). Mmp1 processing of the PDF neuropeptide regulates circadian structural plasticity of pacemaker neurons. PLOS Genet. 10:e1004700. doi: 10.1371/journal.pgen.1004700

Derlig, K., Gießl, A., Brandstätter, J. H., Enz, R., and Dahlhaus, R. (2013). Identification and characterisation of simiate, a novel protein linked to the fragile X syndrome. PLOS ONE 8:e83007. doi: 10.1371/journal.pone.0083007

Dolan, B. M., Duron, S. G., Campbell, D. A., Vollrath, B., Rao, B. S. S., Ko, H.-Y., et al. (2013). Rescue of fragile X syndrome phenotypes in Fmrl KO mice by the small-molecule PAK inhibitor FRAX486. Proc. Natl. Acad. Sci. U.S.A. 110, 5671-5676. doi: 10.1073/pnas.1219383110

Doll, C. A., and Broadie, K. (2014). Impaired activity-dependent neural circuit assembly and refinement in autism spectrum disorder genetic models. Front. Cell. Neurosci. 8:30. doi: 10.3389/fncel.2014.00030

Doll, C. A., and Broadie, K. (2015). Activity-dependent FMRP requirements in development of the neural circuitry of learning and memory. Development 142 , 1346-1356. doi: 10.1242/dev.117127

Doll, C. A., and Broadie, K. (2016). Neuron class-specific requirements for Fragile $\mathrm{X}$ mental retardation protein in critical period development of calcium signaling in learning and memory circuitry. Neurobiol. Dis. 89, 76-87. doi: 10.1016/j.nbd.2016.02.006

Doll, C. A., Vita, D. J., and Broadie, K. (2017). Fragile X mental retardation protein requirements in activity-dependent critical period neural circuit refinement. Curr. Biol. 27, 2318-2330. doi: 10.1016/j.cub.2017.06.046

Fagiolini, M., and Hensch, T. K. (2000). Inhibitory threshold for critical-period activation in primary visual cortex. Nature 404, 183-186. doi: 10.1038/350 04582

Feinberg, E. H., VanHoven, M. K., Bendesky, A., Wang, G., Fetter, R. D., Shen, K., et al. (2008). GFP reconstitution across synaptic partners (GRASP) defines cell contacts and synapses in living nervous systems. Neuron 57, 353-363. doi: 10.1016/j.neuron.2007.11.030

Feng, Y., Gutekunst, C. A., Eberhart, D. E., Yi, H., Warren, S. T., and Hersch, S. M. (1997). Fragile X mental retardation protein: nucleocytoplasmic shuttling and association with somatodendritic ribosomes. J. Neurosci. 17, 1539-1547.

Fernandez, E., Li, K. W., Rajan, N., De Rubeis, S., Fiers, M., Smit, A. B., et al. (2015). FXR2P exerts a positive translational control and is required for the activity-dependent increase of PSD95 expression. J. Neurosci. 35, 9402-9408. doi: 10.1523/JNEUROSCI.4800-14.2015

Friedman, S. H., Dani, N., Rushton, E., and Broadie, K. (2013). Fragile X mental retardation protein regulates trans-synaptic signaling in Drosophila. Dis. Model. Mech. 6, 1400-1413. doi: 10.1242/dmm.012229

Gatto, C. L., and Broadie, K. (2008). Temporal requirements of the fragile X mental retardation protein in the regulation of synaptic structure. Development 135 , 2637-2648. doi: 10.1242/dev.022244

Gatto, C. L., and Broadie, K. (2009). Temporal requirements of the fragile x mental retardation protein in modulating circadian clock circuit synaptic architecture. Front. Neural Circuits 3:8. doi: 10.3389/neuro.04.008.2009 
Gatto, C. L., Pereira, D., and Broadie, K. (2014). GABAergic circuit dysfunction in the Drosophila Fragile X syndrome model. Neurobiol. Dis. 65, 142-159. doi: 10.1016/j.nbd.2014.01.008

Gibson, J. R., Bartley, A. F., Hays, S. A., and Huber, K. M. (2008). Imbalance of neocortical excitation and inhibition and altered UP states reflect network hyperexcitability in the mouse model of fragile X syndrome. J. Neurophysiol. 100, 2615-2626. doi: 10.1152/jn.90752.2008

Goto, A., Kamioka, Y., and Matsuda, M. (2014). PKA modulation of Rac in neuronal cells. Front. Cell. Neurosci. 8:321. doi: 10.3389/fncel.2014.00321

Goto, A., Sumiyama, K., Kamioka, Y., Nakasyo, E., Ito, K., Iwasaki, M., et al. (2013). GDNF and endothelin 3 regulate migration of enteric neural crestderived cells via protein kinase A and Rac1. J. Neurosci. 33, 4901-4912. doi: 10.1523/JNEUROSCI.4828-12.2013

Guven-Ozkan, T., and Davis, R. L. (2014). Functional neuroanatomy of Drosophila olfactory memory formation. Learn. Mem. 21, 519-526. doi: 10.1101/lm. 034363.114

Hamada, F. N., Rosenzweig, M., Kang, K., Pulver, S. R., Ghezzi, A., Jegla, T. J., et al. (2008). An internal thermal sensor controlling temperature preference in Drosophila. Nature 454, 217-220. doi: 10.1038/nature07001

Hanani, M. (2012). Lucifer yellow - an angel rather than the devil. J. Cell. Mol. Med. 16, 22-31. doi: 10.1111/j.1582-4934.2011.01378.x

Harlow, E. G., Till, S. M., Russell, T. A., Wijetunge, L. S., Kind, P., and Contractor, A. (2010). Critical period plasticity is disrupted in the barrel cortex of fmr1 knockout mice. Neuron 65, 385-398. doi: 10.1016/j.neuron.2010.01.024

Harris, K. P., and Littleton, J. T. (2015). Transmission, development, and plasticity of synapses. Genetics 201, 345-375. doi: 10.1534/genetics.115.176529

Henne, W. M., Stenmark, H., and Emr, S. D. (2013). Molecular mechanisms of the membrane sculpting ESCRT pathway. Cold Spring Harb. Perspect. Biol. 5:a016766. doi: 10.1101/cshperspect.a016766

Hensch, T. K. (2004). Critical period regulation. Annu. Rev. Neurosci. 27, 549-579. doi: 10.1146/annurev.neuro.27.070203.144327

Hodges, J. L., Yu, X., Gilmore, A., Bennett, H., Tjia, M., Perna, J. F., et al. (2017). Astrocytic contributions to synaptic and learning abnormalities in a mouse model of fragile X syndrome. Biol. Psychiatry 82, 139-149. doi: 10.1016/j. biopsych.2016.08.036

Huber, K. M., Gallagher, S. M., Warren, S. T., and Bear, M. F. (2002). Altered synaptic plasticity in a mouse model of fragile X mental retardation. Proc. Natl. Acad. Sci. U.S.A. 99, 7746-7750. doi: 10.1073/pnas.122205699

Ito, K., Shinomiya, K., Ito, M., Armstrong, J. D., Boyan, G., Hartenstein, V., et al. (2014). A systematic nomenclature for the insect brain. Neuron 81, 755-765. doi: 10.1016/j.neuron.2013.12.017

Jacobs, S., Cheng, C., and Doering, L. C. (2016). Hippocampal neuronal subtypes develop abnormal dendritic arbors in the presence of Fragile $\mathrm{X}$ astrocytes. Neuroscience 324, 202-217. doi: 10.1016/j.neuroscience.2016.03.011

Jenett, A., Rubin, G. M., Ngo, T.-T. B., Shepherd, D., Murphy, C., Dionne, H., et al. (2012). A GAL4-driver line resource for Drosophila neurobiology. Cell Rep. 2, 991-1001. doi: 10.1016/j.celrep.2012.09.011

Johnson, K. G., Tenney, A. P., Ghose, A., Duckworth, A. M., Higashi, M. E., Parfitt, K., et al. (2006). The HSPGs syndecan and dallylike bind the receptor phosphatase LAR and exert distinct effects on synaptic development. Neuron 49, 517-531. doi: 10.1016/j.neuron.2006.01.026

Kamimura, K., Ueno, K., Nakagawa, J., Hamada, R., Saitoe, M., and Maeda, N. (2013). Perlecan regulates bidirectional Wnt signaling at the Drosophila neuromuscular junction. J. Cell Biol. 200, 219-233. doi: 10.1083/jcb.201207036

Kanellopoulos, A. K., Semelidou, O., Kotini, A. G., Anezaki, M., and Skoulakis, E. M. C. (2012). Learning and memory deficits consequent to reduction of the fragile $\mathrm{x}$ mental retardation protein result from metabotropic glutamate receptor-mediated inhibition of cAMP signaling in Drosophila. J. Neurosci. 32, 13111-13124. doi: 10.1523/JNEUROSCI.1347-12.2012

Kelley, D. J., Davidson, R. J., Elliott, J. L., Lahvis, G. P., Yin, J. C. P., and Bhattacharyya, A. (2007). The cyclic AMP cascade is altered in the fragile X nervous system. PLOS ONE 2:e931. doi: 10.1371/journal.pone.0000931

Kennedy, T., and Broadie, K. (2017). Fragile X mental retardation protein restricts small dye iontophoresis entry into central neurons. J. Neurosci. 37, 9844-9858. doi: 10.1523/JNEUROSCI.0723-17.2017

Kenny, P., and Ceman, S. (2016). RNA secondary structure modulates FMRP's bi-functional role in the MicroRNA pathway. Int. J. Mol. Sci. 17, 1-13. doi: $10.3390 /$ ijms 17060985
Kenny, P. J., Zhou, H., Kim, M., Skariah, G., Khetani, R. S., Drnevich, J., et al. (2014). MOV10 and FMRP regulate AGO2 association with microRNA recognition elements. Cell Rep. 9, 1729-1741. doi: 10.1016/j.celrep.2014. 10.054

Kerr, K. S., Fuentes-Medel, Y., Brewer, C., Barria, R., Ashley, J., Abruzzi, K. C., et al. (2014). Glial Wingless/Wnt regulates glutamate receptor clustering and synaptic physiology at the Drosophila neuromuscular junction. J. Neurosci. 34, 2910-2920. doi: 10.1523/JNEUROSCI.3714-13.2014

Klein, P. S., and Melton, D. A. (1996). A molecular mechanism for the effect of lithium on development. Proc. Natl. Acad. Sci. U.S.A. 93, 8455-8459. doi: $10.1073 /$ pnas.93.16.8455

Koles, K., and Budnik, V. (2012). Wnt signaling in neuromuscular junction development. Cold Spring Harb. Perspect. Biol. 4:a008045. doi: 10.1101/ cshperspect.a008045

Kopke, D. L., Lima, S. C., Alexandre, C., and Broadie, K. (2017). Notum coordinates synapse development via extracellular regulation of Wingless trans-synaptic signaling. Development 144, 3499-3510. doi: 10.1242/dev.148130

Kudumala, S., Freund, J., Hortsch, M., and Godenschwege, T. A. (2013). Differential effects of human L1CAM mutations on complementing guidance and synaptic defects in Drosophila melanogaster. PLOS ONE 8:e76974. doi: 10.1371/journal.pone.0076974

Kuo, C. T., Jan, L. Y., and Jan, Y. N. (2005). Dendrite-specific remodeling of Drosophila sensory neurons requires matrix metalloproteases, ubiquitinproteasome, and ecdysone signaling. Proc. Natl. Acad. Sci. U.S.A. 102, 15230-15235. doi: 10.1073/pnas.0507393102

LaFever, K. S., Wang, X., Page-McCaw, P., Bhave, G., and Page-McCaw, A. (2017). Both Drosophila matrix metalloproteinases have released and membranetethered forms but have different substrates. Sci. Rep. 7:44560. doi: 10.1038/ srep44560

Laggerbauer, B., Ostareck, D., Keidel, E. M., Ostareck-Lederer, A., and Fischer, U. (2001). Evidence that fragile X mental retardation protein is a negative regulator of translation. Hum. Mol. Genet. 10, 329-338. doi: 10.1093/hmg/10.4.329

Lapper, S. R., and Bolam, J. P. (1991). The anterograde and retrograde transport of neurobiotin in the central nervous system of the rat: comparison with biocytin. J. Neurosci. Methods 39, 163-174. doi: 10.1016/0165-0270(91)90082-B

Lee, A., Li, W., Xu, K., Bogert, B. A., Su, K., and Gao, F.-B. (2003). Control of dendritic development by the Drosophila fragile X-related gene involves the small GTPase Rac1. Development 130, 5543-5552. doi: 10.1242/dev.00792

Lee, D. (2015). Global and local missions of cAMP signaling in neural plasticity, learning, and memory. Front. Pharmacol. 6:161. doi: 10.3389/fphar.2015.00161

Lee, J. Y., Geng, J., Lee, J., Wang, A. R., and Chang, K. T. (2017). Activity-induced synaptic structural modifications by an activator of integrin signaling at the Drosophila neuromuscular junction. J. Neurosci. 37, 3246-3263. doi: 10.1523/ JNEUROSCI.3128-16.2017

Lee, L. H., and Godenschwege, T. A. (2015). Structure-function analyses of tyrosine phosphatase PTP69D in giant fiber synapse formation of Drosophila. Mol. Cell. Neurosci. 64, 24-31. doi: 10.1016/j.mcn.2014.11.002

Li, J., Pelletier, M. R., Perez Velazquez, J.-L., and Carlen, P. L. (2002). Reduced cortical synaptic plasticity and GluR1 expression associated with fragile X mental retardation protein deficiency. Mol. Cell. Neurosci. 19, 138-151. doi: 10.1006/mcne.2001.1085

Li, Z., Zhang, Y., Ku, L., Wilkinson, K. D., Warren, S. T., and Feng, Y. (2001). The fragile $\mathrm{X}$ mental retardation protein inhibits translation via interacting with mRNA. Nucleic Acids Res. 29, 2276-2283. doi: 10.1093/nar/29.11.2276

Llano, E., Adam, G., Pendás, A. M., Quesada, V., Sánchez, L. M., Santamaría, I., et al. (2002). Structural and enzymatic characterization of Drosophila Dm2-MMP, a membrane-bound matrix metalloproteinase with tissuespecific expression. J. Biol. Chem. 277, 23321-23329. doi: 10.1074/jbc.M2001 21200

Llano, E., Pendás, A. M., Aza-Blanc, P., Kornberg, T. B., and López-Otín, C. (2000). Dm1 -MMP, a matrix metalloproteinase from Drosophila with a potential role in extracellular matrix remodeling during neural development. J. Biol. Chem. 275, 35978-35985. doi: 10.1074/jbc.M006045200

Logan, M. A. (2017). Fragile phagocytes: FMRP positively regulates engulfment activity. J. Cell Biol. 216, 531-533. doi: 10.1083/jcb.201702034

Lu, T.-Y., MacDonald, J. M., Neukomm, L. J., Sheehan, A. E., Bradshaw, R., Logan, M. A., et al. (2017). Axon degeneration induces glial responses through DraperTRAF4-JNK signalling. Nat. Commun. 8:14355. doi: 10.1038/ncomms14355 
Majumder, P., Chu, J.-F., Chatterjee, B., Swamy, K. B. S., and Shen, C.-K. J. (2016). Co-regulation of mRNA translation by TDP- 43 and fragile $X$ syndrome protein FMRP. Acta Neuropathol. 132, 721-738. doi: 10.1007/s00401-0161603-8

Martinez, V. G., Javadi, C. S., Ngo, E., Ngo, L., Lagow, R. D., and Zhang, B. (2007). Age-related changes in climbing behavior and neural circuit physiology in Drosophila. Dev. Neurobiol. 67, 778-791. doi: 10.1002/dneu. 20388

McBride, S. M. J., Choi, C. H., Wang, Y., Liebelt, D., Braunstein, E., Ferreiro, D., et al. (2005). Pharmacological rescue of synaptic plasticity, courtship behavior, and mushroom body defects in a Drosophila model of fragile X syndrome. Neuron 45, 753-764. doi: 10.1016/j.neuron.2005.01.038

Miller, C. M., Liu, N., Page-McCaw, A., and Broihier, H. T. (2011). Drosophila Mmp2 regulates the matrix molecule faulty attraction (Frac) to promote motor axon targeting in Drosophila. J. Neurosci. 31, 5335-5347. doi: 10.1523/ JNEUROSCI.4811-10.2011

Miller, C. M., Page-McCaw, A., and Broihier, H. T. (2007). Matrix metalloproteinases promote motor axon fasciculation in the Drosophila embryo. Development 135, 95-109. doi: 10.1242/dev.011072

Mines, M. A., and Jope, R. S. (2011). Glycogen synthase kinase-3: a promising therapeutic target for fragile X syndrome. Front. Mol. Neurosci. 4:35. doi: 10.3389/fnmol.2011.00035

Muddashetty, R. S., Kelic, S., Gross, C., Xu, M., and Bassell, G. J. (2007). Dysregulated metabotropic glutamate receptor-dependent translation of AMPA receptor and postsynaptic density-95 mRNAs at synapses in a mouse model of fragile X syndrome. J. Neurosci. 27, 5338-5348. doi: 10.1523/JNEUROSCI.093707.2007

Münch, D., and Galizia, C. G. (2016). DoOR 2.0 - Comprehensive mapping of Drosophila melanogaster odorant responses. Sci. Rep. 6:21841. doi: 10.1038/ srep21841

Musashe, D. T., Purice, M. D., Speese, S. D., Doherty, J., and Logan, M. A. (2016). Insulin-like signaling promotes glial phagocytic clearance of degenerating axons through regulation of draper. Cell Rep. 16, 1838-1850. doi: 10.1016/j. celrep.2016.07.022

Muthukumar, A. K., Stork, T., and Freeman, M. R. (2014). Activity-dependent regulation of astrocyte GAT levels during synaptogenesis. Nat. Neurosci. 17, 1340-1350. doi: 10.1038/nn.3791

Nagappan-Chettiar, S., Johnson-Venkatesh, E. M., and Umemori, H. (2017). Activity-dependent proteolytic cleavage of cell adhesion molecules regulates excitatory synaptic development and function. Neurosci. Res. 116, 60-69. doi: 10.1016/j.neures.2016.12.003

O’Connor, R. M., Stone, E. F., Wayne, C. R., Marcinkevicius, E. V., Ulgherait, M., Delventhal, R., et al. (2017). A Drosophila model of Fragile X syndrome exhibits defects in phagocytosis by innate immune cells. J. Cell Biol. 216, 595-605. doi: $10.1083 /$ jcb. 201607093

Olmos-Serrano, J. L., Paluszkiewicz, S. M., Martin, B. S., Kaufmann, W. E., Corbin, J. G., and Huntsman, M. M. (2010). Defective GABAergic neurotransmission and pharmacological rescue of neuronal hyperexcitability in the amygdala in a mouse model of fragile X syndrome. J. Neurosci. 30, 9929-9938. doi: 10.1523/ JNEUROSCI.1714-10.2010

Pan, L., and Broadie, K. S. (2007). Drosophila fragile X mental retardation protein and metabotropic glutamate receptor a convergently regulate the synaptic ratio of ionotropic glutamate receptor subclasses. J. Neurosci. 27, 12378-12389. doi: 10.1523/JNEUROSCI.2970-07.2007

Pan, L., Woodruff, E. III, Liang, P., and Broadie, K. (2008). Mechanistic relationships between Drosophila fragile X mental retardation protein and metabotropic glutamate receptor A signaling. Mol. Cell. Neurosci. 37, 747-760. doi: 10.1016/j.mcn.2008.01.003

Pan, L., Zhang, Y. Q., Woodruff, E., and Broadie, K. (2004). The Drosophila fragile $\mathrm{X}$ gene negatively regulates neuronal elaboration and synaptic differentiation. Curr. Biol. 14, 1863-1870. doi: 10.1016/j.cub.2004.09.085

Park, P. W., Reizes, O., and Bernfield, M. (2000). Cell surface heparan sulfate proteoglycans: selective regulators of ligand-receptor encounters. J. Biol. Chem. 275, 29923-29926. doi: 10.1074/jbc.R000008200

Pech, U., Pooryasin, A., Birman, S., and Fiala, A. (2013). Localization of the contacts between Kenyon cells and aminergic neurons in the Drosophila melanogaster brain using splitGFP reconstitution. J. Comp. Neurol. 521, 3992-4026. doi: 10.1002/cne.23388
Phelan, P., Nakagawa, M., Wilkin, M. B., Moffat, K. G., O’Kane, C. J., Davies, J. A., et al. (1996). Mutations in shaking-B prevent electrical synapse formation in the Drosophila giant fiber system. J. Neurosci. 16, 1101-1113.

Pulver, S. R., Pashkovski, S. L., Hornstein, N. J., Garrity, P. A., and Griffith, L. C. (2009). Temporal dynamics of neuronal activation by channelrhodopsin-2 and TRPA1 determine behavioral output in Drosophila larvae. J. Neurophysiol. 101, 3075-3088. doi: 10.1152/jn.00071.2009

Reeve, S. P., Bassetto, L., Genova, G. K., Kleyner, Y., Leyssen, M., Jackson, F. R., et al. (2005). The Drosophila fragile X mental retardation protein controls actin dynamics by directly regulating profilin in the brain. Curr. Biol. 15, 1156-1163. doi: 10.1016/j.cub.2005.05.050

Repicky, S., and Broadie, K. (2008). Metabotropic glutamate receptor-mediated use-dependent down-regulation of synaptic excitability involves the fragile $\mathrm{X}$ mental retardation protein. J. Neurophysiol. 101, 672-687. doi: 10.1152/jn. 90953.2008

Santoro, M. R., Bray, S. M., and Warren, S. T. (2012). Molecular mechanisms of fragile X syndrome: a twenty-year perspective. Annu. Rev. Pathol. 7, 219-245. doi: 10.1146/annurev-pathol-011811-132457

Sassone-Corsi, P. (2012). The cyclic AMP pathway. Cold Spring Harb. Perspect. Biol. 4:a011148. doi: 10.1101/cshperspect.a011148

Schenck, A., Bardoni, B., Langmann, C., Harden, N., Mandel, J.-L., and Giangrande, A. (2003). CYFIP/Sra-1 controls neuronal connectivity in Drosophila and links the Rac1 GTPase pathway to the fragile X protein. Neuron 38, 887-898. doi: 10.1016/S0896-6273(03)00354-4

Shilts, J., and Broadie, K. (2017). Secreted tissue inhibitor of matrix metalloproteinase restricts trans -synaptic signaling to coordinate synaptogenesis. J. Cell Sci. 130, 2344-2358. doi: 10.1242/jcs.200808

Silbering, A. F., Rytz, R., Grosjean, Y., Abuin, L., Ramdya, P., Jefferis, G. S. X. E., et al. (2011). Complementary function and integrated wiring of the evolutionarily distinct Drosophila olfactory subsystems. J. Neurosci. 31, 13357-13375. doi: 10.1523/JNEUROSCI.2360-11.2011

Siller, S. S., and Broadie, K. (2011). Neural circuit architecture defects in a Drosophila model of Fragile X syndrome are alleviated by minocycline treatment and genetic removal of matrix metalloproteinase. Dis. Model. Mech. 4, 673-685. doi: 10.1242/dmm.008045

Skoulakis, E. M. C., Kalderon, D., and Davis, R. L. (1993). Preferential expression in mushroom bodies of the catalytic subunit of protein kinase A and its role in learning and memory. Neuron 11, 197-208. doi: 10.1016/0896-6273(93)9 0178-T

Sorkin, A. (1998). Endocytosis and intracellular sorting of receptor tyrosine kinases. Front. Biosci. 3, d729-d738. doi: 10.2741/A316

Stambolic, V., Ruel, L., and Woodgett, J. R. (1996). Lithium inhibits glycogen synthase kinase-3 activity and mimics Wingless signalling in intact cells. Curr. Biol. 6, 1664-1669. doi: 10.1016/S0960-9822(02)70790-2

Sweeney, N. T., Brenman, J. E., Jan, Y. N., and Gao, F.-B. (2006). The coiled-coil protein shrub controls neuronal morphogenesis in Drosophila. Curr. Biol. 16, 1006-1011. doi: 10.1016/j.cub.2006.03.067

Takesian, A. E., and Hensch, T. K. (2013). "Balancing plasticity/stability across brain development," in Progress in Brain Research, eds M. M. Merzenich, M. Nahum, and T. M. Van Vleet (Amsterdam: Elsevier), 3-34. doi: 10.1016/B9780-444-63327-9.00001-1

Tasdemir-Yilmaz, O. E., and Freeman, M. R. (2014). Astrocytes engage unique molecular programs to engulf pruned neuronal debris from distinct subsets of neurons. Genes Dev. 28, 20-33. doi: 10.1101/gad.229518.113

Teis, D., Saksena, S., and Emr, S. D. (2008). Ordered assembly of the ESCRT-III complex on endosomes is required to sequester cargo during MVB formation. Dev. Cell 15, 578-589. doi: 10.1016/j.devcel.2008.08.013

Tejada-Simon, M. V. (2015). Modulation of actin dynamics by Racl to target cognitive function. J. Neurochem. 133, 767-779. doi: 10.1111/jnc.13100

Tessier, C. R., and Broadie, K. (2008). Drosophila fragile X mental retardation protein developmentally regulates activity-dependent axon pruning. Development 135, 1547-1557. doi: 10.1242/dev.015867

Tessier, C. R., and Broadie, K. (2011). The fragile X mental retardation protein developmentally regulates the strength and fidelity of calcium signaling in Drosophila mushroom body neurons. Neurobiol. Dis. 41, 147-159. doi: 10.1016/ j.nbd.2010.09.002

Tessier, C. R., and Broadie, K. (2012). "Molecular and genetic analysis of the Drosophila model of fragile X syndrome," in Results and Problems in Cell 
Differentiation, ed. R. B. Denman (Berlin: Springer), 119-156. doi: 10.1007/9783-642-21649-7_7

Tocchi, A., and Parks, W. C. (2013). Functional interactions between matrix metalloproteinases and glycosaminoglycans. FEBS J. 280, 2332-2341. doi: $10.1111 /$ febs. 12198

Todd, P. K., Mack, K. J., and Malter, J. S. (2003). The fragile X mental retardation protein is required for type-I metabotropic glutamate receptor-dependent translation of PSD-95. Proc. Natl. Acad. Sci. U.S.A. 100, 14374-14378. doi: $10.1073 /$ pnas. 2336265100

Tsai, P.-I., Wang, M., Kao, H.-H., Cheng, Y.-J., Lin, Y.-J., Chen, R.-H., et al. (2012). Activity-dependent retrograde laminin A signaling regulates synapse growth at Drosophila neuromuscular junctions. Proc. Natl. Acad. Sci. U.S.A. 109, 17699-17704. doi: 10.1073/pnas.1206416109

Vita, D. J., and Broadie, K. (2017). ESCRT-III membrane trafficking misregulation contributes to fragile $\mathrm{X}$ syndrome synaptic defects. Sci. Rep. 7:8683. doi: 10.1038/s41598-017-09103-6

Weiler, I. J., Irwin, S. A., Klintsova, A. Y., Spencer, C. M., Brazelton, A. D., Miyashiro, K., et al. (1997). Fragile X mental retardation protein is translated near synapses in response to neurotransmitter activation. Proc. Natl. Acad. Sci. U.S.A. 94, 5395-5400. doi: 10.1073/pnas.94.10.5395

Wlodarczyk, J., Mukhina, I., Kaczmarek, L., and Dityatev, A. (2011). Extracellular matrix molecules, their receptors, and secreted proteases in synaptic plasticity. Dev. Neurobiol. 71, 1040-1053. doi: 10.1002/dneu. 20958

Yan, D., Wu, Y., Feng, Y., Lin, S.-C., and Lin, X. (2009). The core protein of glypican dally-like determines its biphasic activity in wingless morphogen signaling. Dev. Cell 17, 470-481. doi: 10.1016/j.devcel.2009.09.001
Yasunaga, K., Kanamori, T., Morikawa, R., Suzuki, E., and Emoto, K. (2010). Dendrite reshaping of adult Drosophila sensory neurons requires matrix metalloproteinase-mediated modification of the basement membranes. Dev. Cell 18, 621-632. doi: 10.1016/j.devcel.2010.02.010

Zhang, H., Wang, Y., Wong, J. J. L., Lim, K.-L., Liou, Y.-C., Wang, H., et al. (2014). Endocytic pathways downregulate the L1-type cell adhesion molecule neuroglian to promote dendrite pruning in Drosophila. Dev. Cell 30, 463-478. doi: 10.1016/j.devcel.2014.06.014

Zhang, Y. Q., Bailey, A. M., Matthies, H. J. G., Renden, R. B., Smith, M. A., Speese, S. D., et al. (2001). Drosophila fragile X-related gene regulates the MAP1B homolog futsch to control synaptic structure and function. Cell 107, 591-603. doi: 10.1016/S0092-8674(01)00589-X

Zhang, Y. Q., Friedman, D. B., Wang, Z., Woodruff, E., Pan, L., O'Donnell, J., et al. (2005). Protein expression profiling of the Drosophila fragile X mutant brain reveals up-regulation of monoamine synthesis. Mol. Cell. Proteomics 4, 278-290. doi: 10.1074/mcp.M400174-MCP200

Conflict of Interest Statement: The authors declare that the research was conducted in the absence of any commercial or financial relationships that could be construed as a potential conflict of interest.

Copyright (c) 2018 Sears and Broadie. This is an open-access article distributed under the terms of the Creative Commons Attribution License (CC BY). The use, distribution or reproduction in other forums is permitted, provided the original author(s) or licensor are credited and that the original publication in this journal is cited, in accordance with accepted academic practice. No use, distribution or reproduction is permitted which does not comply with these terms. 